\title{
Financial Risk Taking in the Presence of Correlated Non- Financial Background Risk
}

DOI:

10.1016/j.jmateco.2020.03.004

\section{Document Version}

Accepted author manuscript

Link to publication record in Manchester Research Explorer

\section{Citation for published version (APA):}

Chiu, W. H. (2020). Financial Risk Taking in the Presence of Correlated Non-Financial Background Risk. Journal of Mathematical Economics, 88, 167-179. https://doi.org/10.1016/j.jmateco.2020.03.004

\section{Published in:}

Journal of Mathematical Economics

\section{Citing this paper}

Please note that where the full-text provided on Manchester Research Explorer is the Author Accepted Manuscript or Proof version this may differ from the final Published version. If citing, it is advised that you check and use the publisher's definitive version.

\section{General rights}

Copyright and moral rights for the publications made accessible in the Research Explorer are retained by the authors and/or other copyright owners and it is a condition of accessing publications that users recognise and abide by the legal requirements associated with these rights.

\section{Takedown policy}

If you believe that this document breaches copyright please refer to the University of Manchester's Takedown Procedures [http://man.ac.uk/04Y6Bo] or contact uml.scholarlycommunications@manchester.ac.uk providing relevant details, so we can investigate your claim.

\section{OPEN ACCESS}




\title{
Financial Risk Taking in the Presence of Correlated Non-Financial Background Risk
}

\author{
W. Henry Chiu \\ Economics, School of Social Sciences \\ The University of Manchester \\ Manchester, M13 9PL, U.K.
}

March 24, 2020

\begin{abstract}
This paper characterizes the stochastic deterioration resulting from taking a zero-mean financial risk in the presence of correlated non-financial background risk. We show in particular that it has an equivalent stochastic order as well as a necessary and sufficient "integral condition" that implies and is implied by a particular sense in which the stochastic deterioration can be decomposed into a "correlation increase" and a "marginal risk increase". We further characterize a measure of aversion to the stochastic deterioration. These characterizations provide for a more general framework for formulating concepts of increases in risk and correlation and for better understanding risk management decisions governed by individuals' attitudes to them.
\end{abstract}

Key Words: non-financial background risk, expectation dependence, portfolio choice, correlation aversion, correlated risks, marginal risk increase.

JEL Classification: D81. 


\section{Introduction}

Economic decision making under uncertainty often takes place in settings where choices about endogenous risks must be made while simultaneously facing one or more exogenous background risks that are not under the control of the decision maker and can potentially be correlated with the endogenous risks. While considerable effort has been devoted to characterizing in the Expected Utility (EU) framework the conditions on the utility function under which changes in independent financial background risk have a definitive effect on risk aversion, ${ }^{1}$ McFadden (1974) argued that individuals tend not to view endogenous risk and background risk as independent or uncorrelated and it has been shown (by, among others, Doherty and Schlesinger (1983) and Tsetlin and Winkler (2005)) that if the endogenous risk is correlated with the uninsurable background risk, well-known results on optimal risk management decisions assuming uncorrelated background risk can be overturned.

The background risk in the presence of which economic decisions are made can moreover be nonfinancial as well as financial, one's health status being an obvious and often-cited example. ${ }^{2}$ The formal analytical framework for decisions in the presence of such background risk is the more general setting of multivariate distributions that encompasses those for multiplicative as well as additive financial background risks ${ }^{3}$ as special cases. Epstein and Tanny (1980) show that extending analysis analogous to that of Rothschild and Stiglitz (1970) to a setting of bivariate distributions yields a particular sense in which a bivariate distribution exhibiting greater correlation than another and a utility function with a negative cross derivative is necessary and sufficient for aversion to such an increase in correlation. More recently, Muller and Scarsini (2012) show that a discrete multi-variate distribution is preferred to another by all expected utility maximizers whose utility functions have non-positive second and cross derivatives if and only if the former can be obtained from the latter by a finite sequence of "simple inframodular transfers". 4 Notwithstanding these and other important contributions on multivariate stochastic dominance ${ }^{5}$ as well as the existing results explicitly addressing financial risk taking in the presence of non-financial background risk, ${ }^{6}$ little is known about the characteristics of the overall

\footnotetext{
${ }^{1}$ Pratt and Zeckhauser (1987), Kimball (1993), Gollier and Pratt (1996), Eeckhoudt, Gollier, and Schlesinger (1996) are prominent examples.

${ }^{2}$ Other examples of pertinent non-financial risks include uncertain weather conditions, risk of non-property crime, and political risks such as uncertain election outcomes and public policy changes.

${ }^{3}$ See Tsetlin and Winkler (2005) for an exposition on the relevance of multiplicative background risks.

${ }^{4}$ A formal definition of this can be found in Section 2.

${ }^{5}$ Among them are Levy and Paroush (1974), Russell and Seo (1978), Atkinson and Bourguignon (1982) and Gravel and Moyes (2012).

${ }^{6}$ Pratt (1988) considers an individual's attitude toward a financial risk in the presence of a non-financial background risk and obtains a preservation result of decreasing risk aversion when the two risks are independent. Finkelshtain,
} 
risk change induced by taking a financial risk in the presence of potentially correlated non-financial background risks. As a result, a decision maker's attitudes towards such risk changes that determine her optimal economic decisions are not well-understood, still less the behavioral implications of the strength of such attitudes.

This paper defines and characterizes bivariate stochastic deteriorations, termed "correlation-increasing marginal risk increase" (CIMRI) and "correlation-decreasing marginal risk increase" (CDMRI). In the case of random vectors with finite support, a bivariate distribution is a CIMRI (CDMRI) of another if and only if the former can be obtained from the latter by a finite sequence of simple CIMRIs (CDMRIs), each of which can itself be decomposed into an "elementary correlation-increasing (-decreasing) transformation" as defined by Epstein and Tanny (1980) and a simple mean-preserving spread as defined by Rothschild and Stiglitz (1970). We show that a CIMRI (CDMRI) is what results from taking a zero-mean financial risk in the presence of a non-financial background risk that is correlated with the financial risk in the fairly weak sense of positive (negative) expectation dependence, as defined by Wright (1987), and is the stochastic deterioration disliked by all EU maximizers who are both correlation-averse (-loving) as defined by Epstein and Tanny (1980) and marginal risk averse, i.e., with a utility function concave in wealth. Furthermore, the stochastic deteriorations CIMRI and CDMRI each have a necessary and sufficient "integral condition" that is shown to imply and be implied by a particular sense in which a CIMRI (CDMRI) can be decomposed into a correlation increase (decrease) and a marginal risk increase. Together with a comprehensive characterization of attitudes towards financial risk-taking in the presence of a correlated non-financial background risk that we also offer, these results in turn provides an intuitive explanation for, as well as a significant generalization of, existing results on optimal choice of endogenous risk in the presence of financial and non-financial background risks. We further characterize a measure of aversion to CIMRI (CDMRI) and the concept of decreasing aversion to CIMRI (CDMRI) and demonstrate their behavioral implications in the contexts of risk management decisions. In short, the definition and characterization of CIMRI and CDMRI provide for a more general framework for formulating concepts of increases in risk and correlation and for better understanding risk management decisions governed by individuals' attitudes to them.

Kella, and Scarsini (1999) show that the bivariate utility function being concave in wealth and exhibiting "correlationaversion" (as defined by Epstein and Tanny (1980)) is necessary and sufficient for aversion to a zero-mean financial risk in the presence of a positively correlated non-financial risk. In the setting of portfolio choice with one riskless and one risky assets and a non-financial background risk, Li (2011) shows that, assuming the two assets have equal expected returns, an individual with a bivariate utility function concave in wealth and exhibiting correlation-aversion will invest in the risky asset if and only if the background risk and the risky asset return are negatively correlated in the sense of expectation dependence as defined by Wright (1987). 
The rest of the paper is organized as follows. Section 2 defines and characterizes the notions of CIMRI and CDMRI. Section 3 characterizes attitudes towards financial risk-taking in the presence of a correlated non-financial background risk and their implications for optimal decisions. Section 4 characterizes a measure of aversion to CIMRI (CDMRI) and the concept of decreasing aversion to CIMRI (CDMRI). Section 5 concludes.

\section{Increases in Marginal Risk and Correlation}

\subsection{Dependent Random Variables}

Let $(S, \mathcal{S}, P)$ be a probability space where $S$ is the set of states of nature, $\mathcal{S}$ is a $\sigma$-algebra of events, and $P$ is a probability measure on $(S, \mathcal{S})$. Random variables, denoted by $\tilde{x}$, $\tilde{y}$, etc., are functions from $S$ to an interval $[-a, a] \subset R$. Unless indicated otherwise, the cumulative distribution function of the random vector $(\tilde{x}, \tilde{y})$ shall be denoted by $F_{\tilde{x}, \tilde{y}}$. The most basic notion of dependence or correlation between two random variables $\tilde{x}$ and $\tilde{y}$ is that based on the covariance $\operatorname{cov}(\tilde{x}, \tilde{y})$. $\tilde{x}$ and $\tilde{y}$ are said to be positively correlated (negatively correlated, uncorrelated) if $\operatorname{cov}(\tilde{x}, \tilde{y})>(<,=) 0$. Wright (1987) puts forward the notion of negative expectation dependence: $\tilde{x}$ is negative expectation dependent on $\tilde{y}$ if $E[\tilde{x} \mid \tilde{y} \leq y] \geq E[\tilde{x}]$ for all $y$. That is, given the truncation $\tilde{y} \leq y$, the expectation of $\tilde{x}$ is revised upward. For a sharper delineation of the results in what follows, negative (positive) expectation dependence shall denote the strict version of the concept as defined by Wright (1987) while the weak version of the concept will be referred to as "non-positive" ("non-negative") expectation dependence and $\tilde{x}$ is defined to be "zero expectation dependent" on $\tilde{y}$ if $\tilde{x}$ is both non-positive and non-negative expectation dependent on $\tilde{y}$.

\section{Definition 1}

(i) $\tilde{x}$ is positive (negative) expectation dependent on $\tilde{y}$ if $E[\tilde{x} \mid \tilde{y} \leq y] \leq(\geq) E[\tilde{x}]$ for all $y$ and the inequality is strict for $y \in Z_{y} \subset[-a, a]$ where $P\left[\tilde{y}^{-1}\left(Z_{y}\right)\right]>0$.

(ii) $\tilde{x}$ is non-negative (non-positive) expectation dependent on $\tilde{y}$ if $E[\tilde{x} \mid \tilde{y} \leq y] \leq(\geq) E[\tilde{x}]$ for all $y$.

(iii) $\tilde{x}$ is zero expectation dependent on $\tilde{y}$ if $E[\tilde{x} \mid \tilde{y}=y]=E[\tilde{x}]$ for all $y$.

The basic definition of expectation dependence and a key characterization of it that follows clearly indicate that it implies but is not implied by two random variables being correlated. 7

\footnotetext{
${ }^{7}$ Expectation dependence is however the weakest among existing stronger notions of dependence. See Wright (1987) for an exposition of these alternative notions of dependence.
} 
Lemma 1 (Wright (1987)) $\tilde{x}$ is positive (negative, zero) expectation dependent on $\tilde{y}$ if and only if $\operatorname{cov}[\tilde{x}, \psi(\tilde{y})]>(<,=) 0$ for all increasing function $\psi$ for which the covariance exists.

To consider situations involving more than two random variables, we introduce the straightforward extension of Wright's concept that follows.

\section{Definition 2}

(i) Conditional on $\tilde{w}, \tilde{x}$ is positive (negative) expectation dependent on $\tilde{y}$ if $E[\tilde{x} \mid \tilde{y} \leq y, \tilde{w}=w] \leq$ $(\geq) E[\tilde{x} \mid \tilde{w}=w]$ for all $y$ and $w$ and there exists $Z_{w} \subset[-a, a]$ such that $P\left[\tilde{w}^{-1}\left(Z_{w}\right)\right]>0$ and, for $w \in Z_{w}$, the inequality is strict for $y \in Z_{y(w)} \subset[-a, a]$ where $P\left[\tilde{y}^{-1}\left(Z_{y(w)}\right)\right]>0$.

(ii) Conditional on $\tilde{w}, \tilde{x}$ is non-negative (non-positive) expectation dependent on $\tilde{y}$ if $E[\tilde{x} \mid \tilde{y} \leq y, \tilde{w}=$ $w] \leq(\geq) E[\tilde{x} \mid \tilde{w}=w]$ for all $y$ and $w$.

(iii) Conditional on $\tilde{w}, \tilde{x}$ is zero expectation dependent on $\tilde{y}$ if $E[\tilde{x} \mid \tilde{y}=y, \tilde{w}=w]=E[\tilde{x} \mid \tilde{w}=w]$ for all $y$ and $w$.

\subsection{Correlation-Increasing (-decreasing) Marginal Risk Increase}

Consider the concepts of a simple Correlation-Increasing Marginal Risk Increase and a simple Correlationdecreasing Marginal Risk Increase defined in a way analogous to how Rothschild and Stiglitz (1970) define a mean-preserving spread (MPS) and how Epstein and Tanny (1980) define an "elementary Correlation-Increasing Transformation", which we will refer to as a "simple Correlation Increase" for brevity.

Definition 3 Let $F(x, y)$ and $G(x, y)$ be the distribution functions of discrete random vectors $(\tilde{x}, \tilde{y})$ and $\left(\tilde{x}^{\prime}, \tilde{y}^{\prime}\right)$ respectively, and $f(x, y)$ and $g(x, y)$ their corresponding probability mass functions.

(i) $G(x, y)$ differs from $F(x, y)$ by a simple Correlation-Increasing Marginal Risk Increase (CIMRI) if $F(x, y)$ and $G(x, y)$ assign identical probabilities except to the points $\left(x_{0}, y_{1}\right),\left(x_{1}, y_{1}\right),\left(x_{2}, y_{2}\right)$, $\left(x_{3}, y_{2}\right)$ where $y_{1} \leq y_{2}, x_{0}<x_{1}<x_{2}<x_{3}$ and the differences in their probabilities $\psi, \xi \in(0,1)$ satisfy:

$$
\begin{aligned}
& g\left(x_{0}, y_{1}\right)-f\left(x_{0}, y_{1}\right)=\psi, g\left(x_{1}, y_{1}\right)-f\left(x_{1}, y_{1}\right)=-\psi, g\left(x_{2}, y_{2}\right)-f\left(x_{2}, y_{2}\right)=-\xi \\
& g\left(x_{3}, y_{2}\right)-f\left(x_{3}, y_{2}\right)=\xi, \text { and } \psi\left(x_{1}-x_{0}\right)=\xi\left(x_{3}-x_{2}\right) .
\end{aligned}
$$


(ii) $G(x, y)$ differs from $F(x, y)$ by a simple Correlation-Decreasing Marginal Risk Increase (CDMRI) if $F(x, y)$ and $G(x, y)$ assign identical probabilities except to the points $\left(x_{0}, y_{2}\right),\left(x_{1}, y_{2}\right),\left(x_{2}, y_{1}\right)$, $\left(x_{3}, y_{1}\right)$ where $y_{1} \leq y_{2}, x_{0}<x_{1}<x_{2}<x_{3}$ and the differences in their probabilities $\psi, \xi \in(0,1)$ satisfy:

$$
\begin{aligned}
& g\left(x_{0}, y_{2}\right)-f\left(x_{0}, y_{2}\right)=\psi, g\left(x_{1}, y_{2}\right)-f\left(x_{1}, y_{2}\right)=-\psi, g\left(x_{2}, y_{1}\right)-f\left(x_{2}, y_{1}\right)=-\xi \\
& g\left(x_{3}, y_{1}\right)-f\left(x_{3}, y_{1}\right)=\xi, \text { and } \psi\left(x_{1}-x_{0}\right)=\xi\left(x_{3}-x_{2}\right) .{ }^{8}
\end{aligned}
$$

(iii) $G(x, y)$ differs from $F(x, y)$ by a simple Marginal Risk Increase (MRI) if $F(x, y)$ and $G(x, y)$ assign identical probabilities except to the points $\left(x_{0}, \bar{y}\right),\left(x_{1}, \bar{y}\right),\left(x_{2}, \bar{y}\right),\left(x_{3}, \bar{y}\right)$ where $x_{0}<x_{1}<x_{2}<x_{3}$ and the differences in their probabilities $\psi, \xi \in(0,1)$ satisfy:

$$
\begin{aligned}
& g\left(x_{0}, \bar{y}\right)-f\left(x_{0}, \bar{y}\right)=-\psi, g\left(x_{2}, \bar{y}\right)-f\left(x_{2}, \bar{y}\right)=\psi, g\left(x_{1}, \bar{y}\right)-f\left(x_{1}, \bar{y}\right)=-\xi \\
& g\left(x_{3}, \bar{y}\right)-f\left(x_{3}, \bar{y}\right)=\xi, \text { and } \psi\left(x_{1}-x_{0}\right)=\xi\left(x_{3}-x_{2}\right)
\end{aligned}
$$

(iv) $G(x, y)$ differs from $F(x, y)$ by a simple Correlation-Decrease $(C D)$ or equivalently $F(x, y)$ differs from $G(x, y)$ by a simple Correlation-Increase $(C I)$ if $F(x, y)$ and $G(x, y)$ assign identical probabilities except to the points $\left(x_{1}, y_{1}\right),\left(x_{2}, y_{1}\right),\left(x_{1}, y_{2}\right),\left(x_{2}, y_{2}\right)$ where $y_{1}<y_{2}, x_{1}<x_{2}$ and the differences in their probabilities satisfy:

$$
\begin{aligned}
& g\left(x_{1}, y_{1}\right)-f\left(x_{1}, y_{1}\right)=-\xi, g\left(x_{2}, y_{1}\right)-f\left(x_{2}, y_{1}\right)=\xi, g\left(x_{2}, y_{2}\right)-f\left(x_{2}, y_{2}\right)=-\xi \\
& g\left(x_{1}, y_{2}\right)-f\left(x_{1}, y_{2}\right)=\xi
\end{aligned}
$$

The definition is illustrated in the diagram that follows. A simple CIMRI (CDMRI) combines a shift of probability mass from a high (low) to a higher (lower) values of $x$ when the value of $y$ is high with one from a low (high) to a lower (higher) values of $x$ when the value of $y$ is low so as to preserve the mean of $x$.

\footnotetext{
${ }^{8}$ Muller and Scarsini's (2012) notions of ultramodular and inframodular transfers can be defined in our setting of bivariate distributions as follows. $G(x, y)$ differs from $F(x, y)$ by a simple ultramodular transfer or equivalently $F(x, y)$ differs from $G(x, y)$ by a simple inframodular transfer if $F(x, y)$ and $G(x, y)$ assign identical probabilities except to the points $\left(x_{0}, y_{1}\right),\left(x_{1}, y_{1}\right),\left(x_{2}, y_{2}\right),\left(x_{3}, y_{2}\right)$ where $y_{0} \leq y_{1} \leq y_{2} \leq y_{3}, x_{0} \leq x_{1} \leq x_{2} \leq x_{3}$ and the differences in their probabilities $\psi, \xi \in(0,1)$ satisfy:$$
g\left(x_{0}, y_{0}\right)-f\left(x_{0}, y_{0}\right)=\psi, g\left(x_{1}, y_{1}\right)-f\left(x_{1}, y_{1}\right)=-\psi, g\left(x_{2}, y_{2}\right)-f\left(x_{2}, y_{2}\right)=-\xi,
$$$$
g\left(x_{3}, y_{3}\right)-f\left(x_{3}, y_{3}\right)=\xi, \psi\left(x_{1}-x_{0}\right)=\xi\left(x_{3}-x_{2}\right) \text { and } \psi\left(y_{1}-y_{0}\right)=\xi\left(y_{3}-y_{2}\right) \text {. }
$$

Our simple CIMRI is clearly the special case of a simple ultramodular transfer where $y_{0}=y_{1}$ and $y_{2}=y_{3}$. Our simple CDMRI on the other hand is neither a simple ultramodular transfer nor a simple inframodular transfer.

It is also clear that $G(x, y)$ differing from $F(x, y)$ by a CIMRI (with $x_{1}-x_{0}=x_{3}-x_{2}$ ) is equivalent to $F(x, y$ ) differing from $G(x, y)$ by a "between-type progressive income transfer" as Gravel and Moyes (2012) define in the context of inequality measurement.
} 

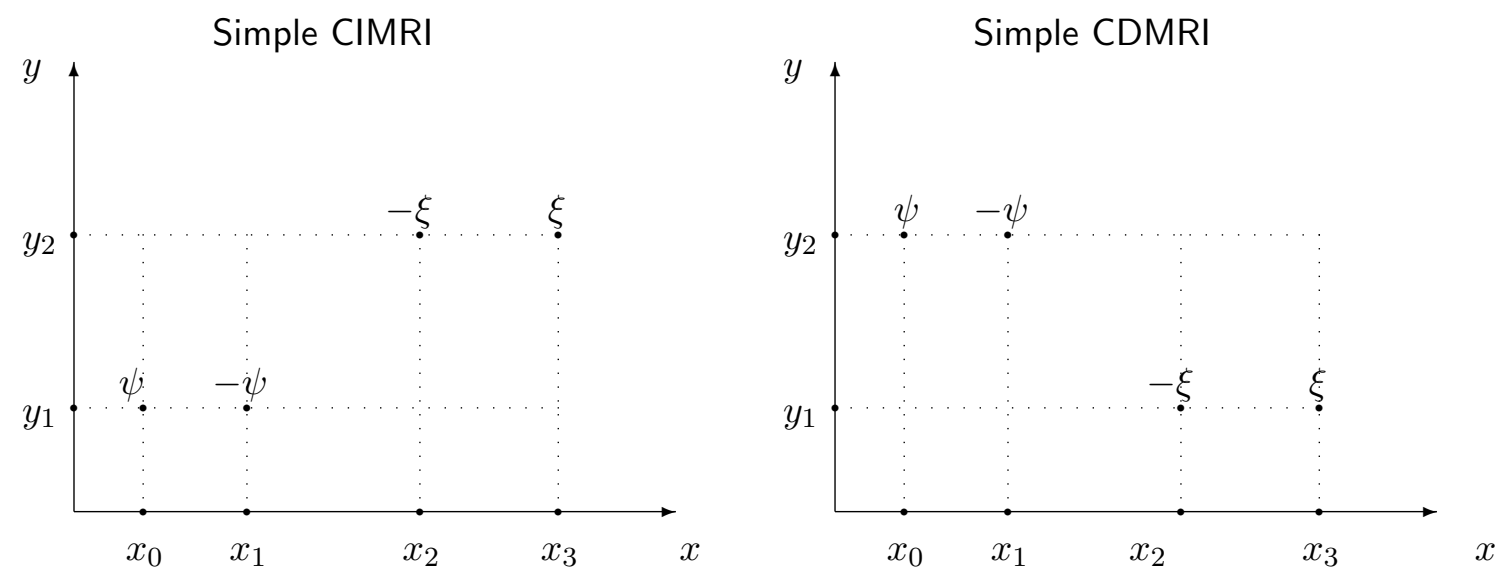

It is clear that a simple MRI is simply an MPS in $x$ in the context of bivariate distributions and it is the special case of a simple CIMRI or CDMRI where $y_{1}=y_{2}$. It is also straightforward to show that a simple CIMRI (CDMRI) can be decomposed into a simple $\mathrm{CI}(\mathrm{CD})$ and a simple MRI as follows. Let $F(x, y)$ and $G(x, y)$ assign identical probabilities except to the points $\left(x_{0}, y_{2}\right),\left(x_{1}, y_{2}\right),\left(x_{2}, y_{1}\right)$, $\left(x_{3}, y_{1}\right)$ where $y_{1} \leq y_{2}, x_{0}<x_{1}<x_{2}<x_{3}$ and the differences in their probabilities $\psi, \xi \in(0,1)$ satisfy:

$$
\begin{aligned}
& g\left(x_{0}, y_{2}\right)-f\left(x_{0}, y_{2}\right)=\psi, g\left(x_{1}, y_{2}\right)-f\left(x_{1}, y_{2}\right)=-\psi, g\left(x_{2}, y_{1}\right)-f\left(x_{2}, y_{1}\right)=-\xi, \\
& g\left(x_{3}, y_{1}\right)-f\left(x_{3}, y_{1}\right)=\xi, \text { and } \psi\left(x_{1}-x_{0}\right)=\xi\left(x_{3}-x_{2}\right)
\end{aligned}
$$

and $F(x, y)$ and $H(x, y)$ assign identical probabilities except to the points $\left(x_{0}, y_{2}\right),\left(x_{1}, y_{2}\right),\left(x_{2}, y_{2}\right)$, $\left(x_{3}, y_{2}\right)$ where the differences in their probabilities $\psi, \eta \in(0,1)$ satisfy:

$$
\begin{aligned}
& h\left(x_{0}, y_{2}\right)-f\left(x_{0}, y_{2}\right)=\psi, h\left(x_{1}, y_{2}\right)-f\left(x_{1}, y_{2}\right)=-\psi, h\left(x_{2}, y_{2}\right)-f\left(x_{2}, y_{2}\right)=-\xi, \\
& h\left(x_{3}, y_{2}\right)-f\left(x_{3}, y_{2}\right)=\xi, \text { and } \psi\left(x_{1}-x_{0}\right)=\xi\left(x_{3}-x_{2}\right) .
\end{aligned}
$$

That is, $G(x, y)$ differs from $F(x, y)$ by a simple CDMRI, and $H(x, y)$ from $F(x, y)$ by a MRI. Then clearly $H(x, y)$ and $G(x, y)$ assign identical probabilities except to the points $\left(x_{2}, y_{1}\right),\left(x_{3}, y_{1}\right),\left(x_{2}, y_{2}\right)$, $\left(x_{3}, y_{2}\right)$ where the differences in their probabilities satisfy:

$$
\begin{aligned}
& g\left(x_{2}, y_{1}\right)-h\left(x_{2}, y_{1}\right)=-\xi, g\left(x_{3}, y_{1}\right)-h\left(x_{3}, y_{1}\right)=\xi, g\left(x_{2}, y_{2}\right)-h\left(x_{2}, y_{2}\right)=\xi, \\
& g\left(x_{3}, y_{2}\right)-h\left(x_{3}, y_{2}\right)=-\xi .
\end{aligned}
$$

That is, $G(x, y)$ differs from $H(x, y)$ by a simple CD. We can thus write

$$
G(x, y)-F(x, y)=[G(x, y)-H(x, y)]+[H(x, y)-F(x, y)]
$$

where $[G(x, y)-H(x, y)]$ is a simple $\mathrm{CD}$ and $[H(x, y)-F(x, y)]$ is an MRI.

We define an EU maximizer to be CIMRI (CDMRI) averse if she dislikes a simple CIMRI (CDMRI).

\section{Definition 4}


(i) $u(x, y)$ is CIMRI averse if $\int_{-a}^{a} u(x, y) d[G(x, y)-F(x, y)] \leq 0$ where $G(x, y)$ differs from $F(x, y)$ by a simple CIMRI or equivalently $\psi u\left(x_{0}, y_{1}\right)+\xi u\left(x_{3}, y_{2}\right) \leq \psi u\left(x_{1}, y_{1}\right)+\xi u\left(x_{2}, y_{2}\right)$ where $y_{1} \leq y_{2}$, $x_{0} \leq x_{1} \leq x_{2} \leq x_{3}$, and $\psi\left(x_{1}-x_{0}\right)=\xi\left(x_{3}-x_{2}\right)$.

(ii) $u(x, y)$ is CDMRI averse if $\int_{-a}^{a} u(x, y) d[G(x, y)-F(x, y)] \leq 0$ where $G(x, y)$ differs from $F(x, y)$ by a simple CDMRI or equivalently $\psi u\left(x_{0}, y_{2}\right)+\xi u\left(x_{3}, y_{1}\right) \leq \psi u\left(x_{1}, y_{2}\right)+\xi u\left(x_{2}, y_{1}\right)$ where $y_{1} \leq y_{2}, x_{0} \leq x_{0} \leq x_{2} \leq x_{3}$, and $\psi\left(x_{1}-x_{0}\right)=\xi\left(x_{3}-x_{2}\right)$.

(iii) $u(x, y)$ is marginal risk averse if $\int_{-a}^{a} u(x, y) d[G(x, y)-F(x, y)] \leq 0$ where $G(x, y)$ differs from $F(x, y)$ by a simple MRI or equivalently $\psi u\left(x_{0}, \bar{y}\right)+\xi u\left(x_{3}, \bar{y}\right) \leq \psi u\left(x_{1}, \bar{y}\right)+\xi u\left(x_{2}, \bar{y}\right)$ where $x_{0} \leq x_{0} \leq x_{2} \leq x_{3}$, and $\psi\left(x_{1}-x_{0}\right)=\xi\left(x_{3}-x_{2}\right)$.

(iv) $u(x, y)$ is correlation averse (loving) if $\int_{-a}^{a} u(x, y) d[G(x, y)-F(x, y)] \leq(\geq) 0$ where $G(x, y)$ differs from $F(x, y)$ by a simple $\mathrm{Cl}$ or equivalently $u\left(x_{1}, y_{1}\right)+u\left(x_{2}, y_{2}\right) \leq(\geq) u\left(x_{1}, y_{2}\right)+$ $u\left(x_{2}, y_{1}\right)$ where $y_{1}<y_{2}$ and $x_{1}<x_{2}$.

Finkelshtain et. al. (1999) show that $u(x, y)$ is submodular and concave in $x$ if and only if $\psi u\left(x_{0}, y_{1}\right)+$ $\xi u\left(x_{3}, y_{2}\right) \leq \psi u\left(x_{1}, y_{1}\right)+\xi u\left(x_{2}, y_{2}\right)$ where $y_{1} \leq y_{2}, x_{0}<x_{1}<x_{2}<x_{3}$, and $\psi\left(x_{1}-x_{0}\right)=\xi\left(x_{3}-x_{2}\right)$, which implies the following.

Lemma 2 In the case where $u$ is differentiable,

(i) $u$ is CIMRI (CDMRI) averse if and only if $u_{11} \leq 0$ and $u_{12} \leq(\geq) 0$.

(ii) $u$ is correlation averse if and only if $u_{12} \leq 0$.

(iii) $u$ is marginal risk averse if and only if $u_{11} \leq 0$.

By transitivity, a CIMRI (CDMRI) averse individual will clearly prefer $F(x, y)$ to $G(x, y)$ if $G(x, y)$ can be obtained from $F(x, y)$ by a finite sequence of simple CIMRIs (CDMRIs), and, given that a simple CIMRI (CDMRI) can be decomposed into a simple $\mathrm{CI}(\mathrm{CD})$ and a simple MRI, an individual is CIMRI (CDMRI) averse if and only if she is both correlation averse (loving) and marginal risk averse.

As a simple MRI is simply an MPS in one of the variables in the context of bivariate distributions, the following characterization of the stochastic deterioration disliked by all marginal risk averse EU maximizers follows immediately from Rothschild and Stiglitz (1970) and Machina and Pratt (1997).

Lemma 3 Let $F(x, y)$ and $G(x, y)$ be the distribution functions of random vectors $(\tilde{x}, \tilde{y})$ and $\left(\tilde{x}^{\prime}, \tilde{y}\right)$ respectively. The following are equivalent. 
(i) $E u(\tilde{x}, \tilde{y}) \geq E u\left(\tilde{x}^{\prime}, \tilde{y}\right)$ for all $u$ such that $u_{11} \leq 0$.

(ii) $\int_{-a}^{x}[G(t \mid \tilde{y}=y)-F(t \mid \tilde{y}=y)] d t \geq 0$ for all $x$ and $y$ and $\int_{-a}^{a}[G(t \mid \tilde{y}=y)-F(t \mid \tilde{y}=y)] d t=0$ for all $y$.

(iii) There exists $\tilde{\epsilon}$ such that $E[\tilde{\epsilon} \mid \tilde{y}=y, \tilde{x}=x]=E[\tilde{\epsilon} \mid \tilde{x}=x]=0$ for all $x$ and $y$ and $F_{\tilde{x}+\tilde{\epsilon}, \tilde{y}}=F_{\tilde{x}^{\prime}}, \tilde{y}$. If $(\tilde{x}, \tilde{y})$ and $\left(\tilde{x}^{\prime}, \tilde{y}\right)$ have finite support, these conditions are also equivalent to

(iv) $G(x, y)$ can be obtained from $F(x, y)$ by a finite sequence of simple MRIs.

Epstein and Tanny (1980), on the other hand, characterize the stochastic deterioration disliked by all correlation-averse EU maximizers as follows.

Lemma 4 Let $F(x, y)$ and $G(x, y)$ be the distribution functions of random vectors $(\tilde{x}, \tilde{y})$ and $\left(\tilde{x}^{\prime}, \tilde{y}\right)$ respectively. The following are equivalent.

(i) $E u(\tilde{x}, \tilde{y}) \geq E u\left(\tilde{x}^{\prime}, \tilde{y}\right)$ for all $u$ such that $u_{12} \leq 0$.

(ii) $G(x, y) \geq F(x, y)$ for all $x$ and $y$ and $G(x, a)=F(x, a)$ for all $x$.

If $(\tilde{x}, \tilde{y})$ and $\left(\tilde{x}^{\prime}, \tilde{y}\right)$ have finite support, these conditions are also equivalent to

(iii) $G(x, y)$ can be obtained from $F(x, y)$ by a finite sequence of simple Cls.

As they are conditions that hold for all random variables whether they have finite support, we use the "integral conditions" to define the notions of a Correlation Increase (Decrease) and a Marginal Risk Increase.

Definition 5 Let $F(x, y)$ and $G(x, y)$ be the distribution functions of random vectors $(\tilde{x}, \tilde{y})$ and $\left(\tilde{x}^{\prime}, \tilde{y}\right)$.

(i) $G(x, y)$ is a Correlation Increase (Cl) of $F(x, y)$ and $F(x, y)$ is a Correlation Decrease (CD) of $G(x, y)$ if $G(x, y) \geq F(x, y)$ for all $x$ and $y$ and $G(x, a)=F(x, a)$ for all $x$.

(ii) $G(x, y)$ is a Marginal Risk Increase (MRI) of $F(x, y)$ if $\int_{-a}^{x}[G(t \mid \tilde{y}=y)-F(t \mid \tilde{y}=y)] d t \geq 0$ for all $x$ and $y$ and $\int_{-a}^{a}[G(t \mid \tilde{y}=y)-F(t \mid \tilde{y}=y)] d t=0$ for all $y$.

Our first main result characterizes the stochastic deterioration disliked by all CIMRI (CDMRI) averse EU maximizers.

Theorem 1 Let $F(x, y)$ and $G(x, y)$ be the distribution functions of random vectors $(\tilde{x}, \tilde{y})$ and $\left(\tilde{x}^{\prime}, \tilde{y}\right)$ respectively. The following are equivalent.

(i) $E u(\tilde{x}, \tilde{y}) \geq E u\left(\tilde{x}^{\prime}, \tilde{y}\right)$ for all $u$ such that $u_{11} \leq 0$ and $u_{12} \leq(\geq) 0$. 
(ii) $\int_{-a}^{a} \int_{-a}^{z(y)}[G(x \mid \tilde{y}=y)-F(x \mid \tilde{y}=y)] d x d F(a, y) \geq 0$ for all $z(y)$ that is non-increasing in $y$ $\left(\int_{-a}^{a} \int_{z(y)}^{a}[G(x \mid \tilde{y}=y)-F(x \mid \tilde{y}=y)] d x d F(a, y) \leq 0\right.$ for all $z(y)$ that is non-decreasing in $\left.y\right)$ and $\int_{-a}^{a}[G(t, a)-F(t, a)] d t=0$.

(iii) There exist $\gamma \in(0,1]$ and distribution functions $\hat{F}(x, y), \hat{G}(x, y)$, and $\hat{H}(x, y)$ such that $\hat{G}(x, y)-$ $\hat{F}(x, y)=\gamma[G(x, y)-F(x, y)], \hat{G}(x, y)$ is a $C l(C D)$ of $\hat{H}(x, y)$ and $\hat{H}(x, y)$ is an MRI of $\hat{F}(x, y)$. If $(\tilde{x}, \tilde{y})$ and $\left(\tilde{x}^{\prime}, \tilde{y}\right)$ have finite support, these conditions are also equivalent to

(iv) $G(x, y)$ can be obtained from $F(x, y)$ by a finite sequence of simple CIMRIs (CDMRIs).

The result shows that the stochastic deterioration from $F(x, y)$ to $G(x, y)$ is disliked by all CIMRI (CDMRI) averse EU maximizers if and only if it can be decomposed into a $\mathrm{Cl}(\mathrm{CD})$ and an $\mathrm{MRI}$ in the sense that there exist distributions $\hat{F}(x, y) \hat{G}(x, y) \hat{H}(x, y)$ such that the shift from $\hat{F}(x, y)$ to $\hat{G}(x, y)$ is "EU-equivalent" to that from $F(x, y)$ to $G(x, y)$, i.e., $\gamma \in(0,1]$ such that $\hat{G}(x, y)-\hat{F}(x, y)=$ $\gamma[G(x, y)-F(x, y)],{ }^{9}$ and $\hat{G}(x, y)$ is a $\mathrm{Cl}$ of $\hat{H}(x, y)$ and $\hat{H}(x, y)$ is an MRI of $\hat{F}(x, y)$. That is, we can write

$$
\hat{G}(x, y)-F(x, y)=[\hat{G}(x, y)-\hat{H}(x, y)]+[\hat{H}(x, y)-\hat{F}(x, y)]
$$

where $[\hat{G}(x, y)-\hat{H}(x, y)]$ is a $\mathrm{CD}$ and $[\hat{H}(x, y)-\hat{F}(x, y)]$ is an MRI. Furthermore, the stochastic deterioration has a necessary and sufficient "integral condition" that can be seen as a generalization of that for a "mean-preserving increase in risk" obtained in Rothschild and Stiglitz (1970) and is equivalent to what Gravel and Moyes (2012) obtain in the setting of inequality measurement where random variables have finite support. In the case of random vectors with finite support, the stochastic deterioration is also equivalent to a finite sequence of simple CIMRIs (CDMRIs).

It is worth noting that, unlike the special case $u(x, y)=u(x+y)$ where $u_{11} \leq 0$ implies $u_{12} \leq 0$, a general bivariate VNM utility function $u(x, y)$ permits $u_{11} \leq 0$ and $u_{12} \geq 0$ as well as $u_{11} \leq 0$ and $u_{12} \leq 0$, i.e., the disentanglement of the attitudes towards marginal risk and correlation. In many applications, including the examples where $y$ represents an individual's health status or weather condition, it is in fact arguable that $u_{11} \leq 0$ and $u_{12} \geq 0$ is the more plausible assumption as individuals who are averse to financial risk (i.e., $u_{11} \leq 0$ ) may regard wealth and health (or pleasant weather conditions) as complements (i.e., $u_{12} \geq 0$ ). ${ }^{10} \mathrm{CDMRI}$ as the stochastic order defined by the preferences of EU

\footnotetext{
${ }^{9}$ Under this condition, $\hat{F}(x, y)$ is preferred to $\hat{G}(x, y)$ by an EU maximizer if and only if $F(x, y)$ is preferred to $G(x, y)$.

${ }^{10}$ Furthermore, Quah (2007, Proposition 1) implies that in the standard two-good consumer problem, the condition $u_{12} \geq 0$, together with $u_{11} \leq 0$ and $u_{22} \leq 0$, on the bivariate utility function $u(x, y)$ is sufficient for demand to be normal.
} 
maximizers with $u_{11} \leq 0$ and $u_{12} \geq 0$, which is neither an inframodular transfer nor an ultramodular transfer as defined by Muller and Scarsini (2012), is thus no less relevant than CIMRI.

As they are conditions that hold for all random variables whether they have finite support, we use the "integral conditions" to define the notions of a Correlation-Increasing Marginal Risk Increase and a Correlation-Decreasing Marginal Risk Increase.

Definition 6 Let $F(x, y)$ and $G(x, y)$ be the distribution functions of random vectors $(\tilde{x}, \tilde{y})$ and $\left(\tilde{x}^{\prime}, \tilde{y}\right)$.

(i) $G(x, y)$ is a Correlation-Increasing Marginal Risk Increase (CIMRI) of F(x,y) if $\int_{-a}^{a} \int_{-a}^{z(y)}[G(x \mid \tilde{y}=y)-F(x \mid \tilde{y}=y)] d x d F(a, y) \geq 0$ for all $z(y)$ that is non-increasing in $y$ and $\int_{-a}^{a}[G(t, a)-F(t, a)] d t=0$.

(ii) $G(x, y)$ is a Correlation-Decreasing Marginal Risk Increase (CDMRI) of F(x,y) if $\int_{-a}^{a} \int_{z(y)}^{a}[G(x \mid \tilde{y}=y)-F(x \mid \tilde{y}=y)] d x d F(a, y) \leq 0$ for all $z(y)$ that is non-decreasing in $y$ and $\int_{-a}^{a}[G(t, a)-F(t, a)] d t=0$.

\section{Aversion to a Financial Risk in the Presence of a Non-financial Background Risk}

Having characterized in the previous section the stochastic orders of CIMRI and CDMRI, we show in this section that a CIMRI (CDMRI) is what results from taking a zero-mean financial risk in the presence of a non-financial background risk that is correlated with the financial risk in the sense of positive (negative) expectation dependence and a comprehensive characterization of attitudes towards such risk taking as well as their implications for optimal decisions can be derived from the behavior of the function $\phi(\alpha)$ defined by

$$
\phi(\alpha) \equiv E u(\tilde{w}+\alpha \tilde{\epsilon}, \tilde{y})
$$

where $E[\tilde{\epsilon} \mid \tilde{w}]=0$. The following result describes the behavior of $\phi(\alpha)$ in its relation to how the risks $\tilde{\epsilon}, \tilde{y}$, and $\tilde{w}$ are correlated.

Theorem 2 Suppose $E[\tilde{\epsilon} \mid \tilde{w}]=0$.

(i) Assume $u_{11}<0$ and $u_{12}<(>) 0 . \phi^{\prime}(\alpha)<0$ for $\alpha \geq 0$ if and only if, conditional on $\tilde{w}, \tilde{\epsilon}$ is positive (negative) expectation dependent on $\tilde{y}$.

(ii) Assume $u_{11}<0$ and $u_{12}<(>) 0 . \phi^{\prime}(\alpha)>0$ for $\alpha \leq 0$ if and only if, conditional on $\tilde{w}, \tilde{\epsilon}$ is negative (positive) expectation dependent on $\tilde{y}$. 
(iii) Assume $u_{11}<0$ and $u_{12}<0$ or $u_{12}>0$. $\phi^{\prime}(\alpha)<0$ for $\alpha>0, \phi^{\prime}(\alpha)>0$ for $\alpha<0$ and $\phi^{\prime \prime}(\alpha)>0$ if and only if $E[\tilde{\epsilon} \mid \tilde{y}=y, \tilde{w}=w]=E[\tilde{\epsilon} \mid \tilde{w}=w]$ for all $w$ and $y$.

(iv) For any $\tilde{w}, \tilde{\epsilon}$ and $\tilde{y}$ such that, conditional on $\tilde{w}, \tilde{\epsilon}$ is non-negative (non-positive) expectation dependent on $\tilde{y}$, and any $\alpha \geq 0, \phi^{\prime}(\alpha) \leq 0$ if and only if $u_{11} \leq 0$ and $u_{12} \leq(\geq) 0$.

(v) For any $\tilde{w}, \tilde{\epsilon}$ and $\tilde{y}$ such that, conditional on $\tilde{w}, \tilde{\epsilon}$ is non-positive (non-negative) expectation dependent on $\tilde{y}$, and any $\alpha \leq 0, \phi^{\prime}(\alpha) \geq 0$ if and only if $u_{11} \leq 0$ and $u_{12} \leq(\geq) 0$.

That is, $u_{11} \leq 0$ and $u_{12} \leq(\geq) 0$ are necessary and sufficient for an EU maximizer to be averse to more of a financial risk $\tilde{\epsilon}$ that, conditional on the financial background risk $\tilde{w}$, has a zero mean and is nonnegative (non-positive) expectation dependent on the non-financial background risk $\tilde{y}$. Furthermore, an EU maximizer who is both strictly marginal risk averse and strictly correlation averse (loving) dislikes more of a financial risk $\tilde{\epsilon}$ in the presence of a financial background risk $\tilde{w}$ and a non-financial background risk $\tilde{y}$ if and only if, conditional on $\tilde{w}, \tilde{\epsilon}$ has a zero mean and is positive (negative) expectation dependent on $\tilde{y}$. In view of Theorem 1 , this implies the following.

Corollary 1 For $\alpha \geq 0$, an increase in $\alpha$ induces a CIMRI (CDMRI, MRI) in $F_{\tilde{w}+\alpha \tilde{\epsilon}, \tilde{y}}$ if $E[\tilde{\epsilon} \mid \tilde{y} \leq y, \tilde{w}=$ $w] \leq(\geq,=) E[\tilde{\epsilon} \mid \tilde{w}=w]=0$ for all $w$ and $y$.

The result identifies, independently of the EU framework, the overall risk change resulting from taking more of a financial risk $\tilde{\epsilon}$ that, conditional on the financial background risk $\tilde{w}$, has a zero mean and is non-negative (non-positive, zero) expectation dependent on the non-financial background risk $\tilde{y}$. This provides for establishing the "order" of aversion to CIMRI, CDMRI, and MRI in what follows.

Letting $\succsim$ denote a utility-representable preference relation on the set of random variables ${ }^{11}$ and $\pi(\alpha)$ denote the risk premium defined by

$$
w-\pi(\alpha) \sim w+\alpha \tilde{\epsilon}
$$

where $E[\tilde{\epsilon}]=0$. Segal and Spivak (1990) define that a decision-maker's risk attitude is first-order if $\pi^{\prime}(0) \neq 0$ for all $\tilde{\epsilon}$ being non-degenerate and is second-order if $\pi^{\prime}(0)=0$ and $\pi^{\prime \prime}(0) \neq 0$ for all $\tilde{\epsilon}$ being non-degenerate and show that, given $E[\tilde{x}]>0$, a decision-maker exhibiting first-order risk aversion (i.e., negative first-order risk attitude) will prefer the sum $w$ with certainty to $(w+\alpha \tilde{x})$ for a sufficiently small

\footnotetext{
${ }^{11}$ That is, $\succsim$ is complete, transitive, and continuous with respect to the topology of weak convergence.
} 
$\alpha$ if $E[\tilde{x}]$ is small enough while one exhibiting second-order risk aversion will prefer $(w+\alpha \tilde{x})$ to $w$ with certainty for a sufficiently small $\alpha$.

Define $\pi_{c}(\alpha)$ by

$$
E u\left(\tilde{w}-\pi_{c}(\alpha), \tilde{y}\right)=E u(\tilde{w}+\alpha \tilde{\epsilon}, \tilde{y})
$$

where $E[\tilde{\epsilon} \mid \tilde{w}]=0$. In the special case where $\tilde{w}$ is degenerate, $\pi_{c}(\alpha)$ is what Dionne and Li (2014) define to be "conditional dependent risk premium". Theorem 2 also implies the following.

\section{Corollary 2}

(i) Assume $u_{11}<0$ and $u_{12}<(>) 0 . \pi_{c}^{\prime}(0)>0$ if $E[\tilde{\epsilon} \mid \tilde{w}]=0$ and, conditional on $\tilde{w}, \tilde{\epsilon}$ is positive (negative) expectation dependent on $\tilde{y}$.

(ii) Assume $u_{11}<0 . \pi_{c}^{\prime}(0)=0$ and $\pi_{c}^{\prime \prime}(0)>0$ if $E[\tilde{\epsilon} \mid \tilde{y}=y, \tilde{w}=w]=E[\tilde{\epsilon} \mid \tilde{w}=w]=0$ for all $w$ and $y$.

(iii) Let $E[\tilde{x} \mid \tilde{w}]=E[\tilde{x}]>0$. If $\pi_{c}^{\prime}(0)>0$ for all $\tilde{\epsilon}$ such that $E[\tilde{\epsilon} \mid \tilde{w}]=0$, then for a sufficiently small $\alpha, E u(\tilde{w}, \tilde{y})>E u(\tilde{w}+\alpha \tilde{x}, \tilde{y})$ if $E[\tilde{x}]$ is small enough.

(iv) Let $E[\tilde{x} \mid \tilde{w}]=E[\tilde{x}]>0$. If $\pi_{c}^{\prime}(0)=0$ and $\pi_{c}^{\prime \prime}(0)>0$ for all $\tilde{\epsilon}$ such that $E[\tilde{\epsilon} \mid \tilde{w}]=0$, then for a sufficiently small $\alpha, E u(\tilde{w}, \tilde{y})<E u(\tilde{w}+\alpha \tilde{x}, \tilde{y})$.

In view of Segal and Spivak (1990), since for $\alpha \geq 0$, an increase in $\alpha$ induces a CIMRI (CDMRI, MRI) in $F_{\tilde{w}+\alpha \tilde{\epsilon}, \tilde{y}}$ if, conditional on $\tilde{w}, \tilde{\epsilon}$ is positive (negative, zero) expectation dependent on $\tilde{y}$ (Corollary 1), Corollary 2 has the interpretation that while aversion to MRI is second-order, aversion to CIMRI (CDMRI) and hence aversion to $\mathrm{Cl}(\mathrm{CD})$ are first-order, with a CIMRI (CDMRI) being a combination of a $\mathrm{Cl}(\mathrm{CD})$ and an MRI.

The characterization of attitudes towards financial risk in the presence of a correlated non-financial background risk established in Theorem 2 further implies regularities on optimal risk management decisions in their relations to financial and non-financial background risks. Consider an EU maximizing agent's decision problem of the following form:

$$
\max _{\alpha} V(\alpha) \equiv E u(\tilde{w}+\alpha \tilde{x}, \tilde{y})
$$

That is, in the presence of a non-financial background risk $\tilde{y}$, the financial payoff of choosing of $\alpha$ is given by $\tilde{w}+\alpha \tilde{x}$. Prominent examples of this class of decision problems include the following. First, 
if $\tilde{w}_{0}$ denotes the random initial wealth and $\alpha$ the amount invested in a risky asset whose random return rate is $\tilde{r}$ and there is a riskless asset whose return rate is $r_{0}$, then the final wealth in this portfolio-choice problem is $\tilde{y}=\left(1+r_{0}\right)\left(\tilde{w}_{0}-\alpha\right)+\alpha(1+\tilde{r})=\left(1+r_{0}\right) \tilde{w}_{0}+\alpha\left(\tilde{r}-r_{0}\right) \equiv \tilde{w}+\alpha \tilde{x}$, where $\tilde{w} \equiv\left(1+r_{0}\right) \tilde{w}_{0}$ and $\tilde{x} \equiv \tilde{r}-r_{0}$. A variant of the problem takes the following form: An investor has to allocate his uncertain initial wealth $\tilde{w}_{0}$ between two assets, which are identified with their uncertain return rates $\tilde{r}$ and $\tilde{r}_{0}$. Letting $\alpha$ be the amount invested in asset $\tilde{r}$, his final wealth is then $\tilde{y}=\left(1+\tilde{r}_{0}\right)\left(\tilde{w}_{0}-\alpha\right)+\alpha(1+\tilde{r})=\left(1+\tilde{r}_{0}\right) \tilde{w}_{0}+\alpha\left(\tilde{r}-\tilde{r}_{0}\right) \equiv \tilde{w}+\alpha \tilde{x}$, where $\tilde{w} \equiv\left(1+\tilde{r}_{0}\right) \tilde{w}_{0}$ and $\tilde{x} \equiv \tilde{r}-\tilde{r}_{0}$. Secondly, let $\tilde{l}$ be the random insurable loss, $\beta$ the coinsurance rate, $\pi=\beta\left(\mu_{\tilde{l}}+\delta\right)$ the insurance premium, and $\tilde{w}_{0}$ still the random initial wealth. Then the final wealth in this insurancepurchasing problem is $\tilde{y}=\tilde{w}_{0}-\tilde{l}+\beta \tilde{l}-\beta\left(\mu_{\tilde{l}}+\delta\right)=\left[\tilde{w}_{0}-\mu_{\tilde{l}}-\delta\right]+(1-\beta)\left(\mu_{\tilde{l}}-\tilde{l}+\delta\right) \equiv \tilde{w}+\alpha \tilde{x}$ where $\tilde{w} \equiv\left[\tilde{w}_{0}-\mu_{\tilde{l}}-\delta\right], \alpha \equiv(1-\beta)$, and $\tilde{x} \equiv\left(\mu_{\tilde{l}}-\tilde{l}+\delta\right)$.

Let $\alpha^{*}$ maximizes $E u(\tilde{w}+\alpha \tilde{x}, \tilde{y})$. Since $V^{\prime \prime}(\alpha)<0$ given $u_{11}<0, \alpha^{*}>(<,=) 0$ if and only if $V^{\prime}(0)>(<,=) 0$. Letting $\tilde{\epsilon}=\tilde{x}-E[\tilde{x}]$, we have

$$
V^{\prime}(0)=E(E[\tilde{x}]+\tilde{\epsilon}) u_{1}(\tilde{w}, \tilde{y})=E[\tilde{x}] E u_{1}(\tilde{w}, \tilde{y})+\phi^{\prime}(0)
$$

Theorem 2 thus implies the following.

Corollary 3 Let $\alpha^{*}$ maximizes $E u(\tilde{w}+\alpha \tilde{x}, \tilde{y})$.

(i) Assuming $u_{11}<0$ and $u_{12}<0$ and $E[\tilde{x} \mid \tilde{w}]=E[\tilde{x}]=0, \alpha^{*}>(<,=) 0$ if and only if, conditional on $\tilde{w}, \tilde{x}$ is negative (positive, zero) expectation dependent on $\tilde{y}$.

(ii) Assuming $u_{11}<0$ and $u_{12}>0$ and $E[\tilde{x} \mid \tilde{w}]=E[\tilde{x}]=0, \alpha^{*}>(<,=) 0$ if and only if, conditional on $\tilde{w}, \tilde{x}$ is positive (negative, zero) expectation dependent on $\tilde{y}$.

(iii) $\alpha^{*}>(<,=) 0$ if $u_{11}<0$ and $u_{12}<0$ and $E[\tilde{x} \mid \tilde{w}]=E[\tilde{x}] \geq(\leq,=) 0$ and, conditional on $\tilde{w}$, $\tilde{x}$ is negative (positive, zero) expectation dependent on $\tilde{y}$.

(iv) $\alpha^{*}>(<,=) 0$ if $u_{11}<0$ and $u_{12}>0$ and $E[\tilde{x} \mid \tilde{w}]=E[\tilde{x}] \geq(\leq,=) 0$ and, conditional on $\tilde{w}$, $\tilde{x}$ is positive (negative, zero) expectation dependent on $\tilde{y}$.

The result, together with Theorem 1 and Corollaries 1 and 2, provides an intuitive explanation for, as well as a generalization of, key results in $\mathrm{Li}(2011)$ where the special case of a degenerate $\tilde{w}$ is considered: In the portfolio choice setting with one risky asset and one riskless asset, if, conditional on $\tilde{w}, \tilde{x}$ has a 
zero mean and is zero expectation dependent on $\tilde{y}$, since investing in the risky asset induces only an MRI, an individual who is strictly marginal risk averse $\left(u_{11}<0\right)$ naturally chooses not to invest in the asset. If, on the other hand, conditional on $\tilde{w}, \tilde{x}$ is negative (positive) expectation dependent on $\tilde{y}$, an individual who is both strictly correlation-averse (loving) and strictly marginal risk averse will choose to invest in the risky asset even if its conditional expected return is zero since investing the asset induces a combination of a correlation decrease (increase) and a marginal risk increase with the effect of the former, which is first-order, dominating that of the latter, which is second-order.

Note that, in the context of health insurance purchasing in the presence of uncertain health status as the background risk, $E[\tilde{x} \mid \tilde{w}]=E[\tilde{x}]=0$ corresponds to actuarially fair insurance and $\alpha^{*}>(<,=) 0$ to choosing partial (over, full) insurance coverage. Furthermore, an insurance policy with proportional premium loadings is one whose premium $(\pi)$ is linear in the coinsurance rate $(\beta=(1-\alpha))$. In this setting, our result implies that if the insurance premium is actuarially fair and, conditional on his initial wealth, an individual's health care cost is negative expectation dependent on his health status, then a CDMRI averse individual will purchase partial health insurance coverage while a CIMRI averse individual will purchase more than full health insurance coverage. On the other hand, with proportional premium loadings, if, conditional on their initial wealth, an individual's health care cost is negative expectation dependent on his health status, while a CIMRI averse individual will purchase partial health insurance coverage, a CDMRI averse individual may still choose full health insurance coverage. These results highlight the contrast between the insurance purchasing behavior of a CDMRI averter and that of a CIMRI averter under the plausible assumption that an individual's health care cost is negative expectation dependent on his health status. Since the insurance purchasing behavior of a risk averter obtained by Doherty and Schlesinger (1983) in a simple univariate setting with correlated financial background risk is analogous to that of a CIMRI averter here and, as argued earlier, individuals who are averse to financial risk (i.e., $u_{11}<0$ ) may regard wealth and health as complements (i.e., $u_{12}>0$ ) and hence are averse to CDMRI, the result illustrates the importance of a more general bivariate-distribution framework where attitudes toward financial risk and correlation can be disentangled.

Given that the general results in Theorem 2 clearly also apply to the special case where $u(x, y)=$ $u(x y)$, it has implications for optimal risk management decisions in the presence of multiplicative background risk. In particular, the condition $u_{12}(x, y)<(>) 0$ for all $x, y$ becomes $u^{\prime}(x y)+x y u^{\prime \prime}(x y)<$ $(>) 0$ for all $x, y$ and is thus equivalent to the condition $u^{\prime}(x)+x u^{\prime \prime}(x)<(>) 0$ for all $x$, which is a key condition in what is obtained in Tsetlin and Winkler (2005) and Li (2011) concerning optimal 
risk management decisions in the presence of multiplicative background risk where the special case of $\tilde{w}$ being degenerate is considered. That is, Theorem 2 offers an intuitive explanation for, as well as a generalization of their results.

Applying the general results in Theorem 2 to the special case where $u(x, y)=u(x+y)$ on the other hand yields a generalization of the results obtained by Doherty and Schlesinger (1983), Tsetlin and Winkler (2005), and Wright (1987) on insurance purchasing and portfolio choice in the presence of an additive financial background risk. ${ }^{12}$

\section{Strength of Aversion to Marginal Risk and Correlation}

The characterization of an individual's attitude toward taking a financial risk in the presence of a correlated non-financial background risk in the previous section leads naturally to the question of how one characterizes the strength of such attitudes and its behavioral implications. We first introduce the following definition.

Definition $7 v$ is more averse to CIMRI $(C D M R I)$ than $u$ if $E u\left(\tilde{x}^{\prime}, \tilde{y}\right) \leq E u(\tilde{x}-\pi, \tilde{y})$ implies $E v\left(\tilde{x}^{\prime}, \tilde{y}\right) \leq$

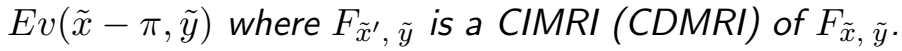

Being more averse to CIMRI (CDMRI) thus means being willing to pay more to avoid a CIMRI (CDMRI).

The result that follows characterizes the concept in terms of the underlying EU preferences as well as its behavioral implications.

Theorem 3 Assume $u_{11} \leq 0$ and $u_{12} \leq(\geq)$. The following are equivalent

(i) $v$ is more averse to CIMRI (CDMRI) than $u$.

(ii) There exists $\lambda>0$ such that for all $x$ and $y$,

$$
\lambda u_{1}(x, y) \geq v_{1}(x, y), \lambda u_{11}(x, y) \geq v_{11}(x, y) \text { and } \lambda u_{12}(x, y) \geq(\leq) v_{12}(x, y)
$$

\footnotetext{
${ }^{12}$ The result on optimal insurance purchasing implied by Theorem 2 essentially shows that the regularities obtained in a simple example assuming EU maximization by Doherty and Schlesinger (1983) can be extended to our much more general setting. In the portfolio choice setting with one risky asset and one riskless asset, Tsetlin and Winkler (2005) show that if the return of the risky asset has a zero mean but is "negatively related" to the background risk, it is optimal to invest a positive amount in the asset, where a random variable $\tilde{x}$ is defined to be negatively related to another $\tilde{y}$ if $E[\tilde{x} \mid \tilde{y}=y]$ is decreasing in $y$. In the setting with non-random initial wealth and two risky assets whose return rates are $\tilde{r}$ and $\tilde{r}_{0}$ respectively, Wright (1987) shows that if $E[\tilde{r}] \geq E\left[\tilde{r}_{0}\right],\left(\tilde{r}-\tilde{r}_{0}\right)$ being negative expectation dependent on $\tilde{r}_{0}$ implies that all risk averse EU maximizers invest a positve amount in the asset with return rate $\tilde{r}$ and if $E[\tilde{r}]=E\left[\tilde{r}_{0}\right]$, then all risk averse EU maximizers invest a positve amount in both assets if and only if $\left(\tilde{r}-\tilde{r}_{0}\right)$ is negative expectation dependent on $\tilde{r}_{0}$ and $\left(\tilde{r}_{0}-\tilde{r}\right)$ is negative expectation dependent on $\tilde{r}$.
} 
(iii) There exist $\Gamma(x, y)$ and $\lambda>0$ such that $\Gamma_{1}(x, y) \leq 0, \Gamma_{11}(x, y) \leq 0, \Gamma_{12}(x, y) \leq(\geq) 0$, and $v(x, y)=\lambda u(x, y)+\Gamma(x, y)$.

(iv) For all $\tilde{w}, \tilde{\epsilon}$, and $\tilde{y}$ such that $E[\tilde{\epsilon} \mid \tilde{y} \leq y, \tilde{w}=x] \leq(\geq) E[\tilde{\epsilon} \mid \tilde{w}=w]=0$ for all $w$ and $y$, $\alpha_{2}>\alpha_{1} \geq 0, E u\left(\tilde{w}+\alpha_{2} \tilde{\epsilon}+\pi^{u}, \tilde{y}\right)=E u\left(\tilde{w}+\alpha_{1} \tilde{\epsilon}, \tilde{y}\right)$ and $E v\left(\tilde{w}+\alpha_{2} \tilde{\epsilon}+\pi^{v}, \tilde{y}\right)=E v\left(\tilde{w}+\alpha_{1} \tilde{\epsilon}, \tilde{y}\right)$ imply that $\pi^{v} \geq \pi^{u}$.

(v) If $\alpha_{u}^{*}(\tilde{w}, \tilde{x}, \tilde{y})$ and $\alpha_{v}^{*}(\tilde{w}, \tilde{x}, \tilde{y})$ respectively maximize $E u(\tilde{w}+\alpha \tilde{x}, \tilde{y})$ and $E v(\tilde{w}+\alpha \tilde{x}, \tilde{y})$, then $\alpha_{u}^{*}(\tilde{w}, \tilde{x}, \tilde{y}) \geq \alpha_{v}^{*}(\tilde{w}, \tilde{x}, \tilde{y})$ for all $\tilde{w}, \tilde{x}$, and $\tilde{y}$ such that $E[\tilde{x} \mid \tilde{y} \leq y, \tilde{w}=w] \leq(\geq) E[\tilde{x} \mid \tilde{w}=w]=$ $E[\tilde{x}]>0$ for all $w$ and $y$.

The result can be seen to be an extension of Machina and Nielson's (1987) 4-way characterization of Ross's (1981) strong measure of risk aversion to the more general setting of bivariate distributions where attitudes toward marginal risk and correlation can be disentangled. In the context of health insurance purchasing in the presence of uncertain health status (as the non-financial background risk), the result says that if, conditional on his initial wealth, an individual's health care cost is non-positive expectation dependent on his health status, then with proportional premium loadings, an individual will purchase more health insurance coverage if and only if he is more averse to CIMRI.

To study the implications of aversion to CIMRI (CDMRI) for individual behavior, we will adopt the following definitions.

\section{Definition 8}

(i) $u$ exhibits decreasing aversion to CIMRI (CDMRI) if $E u\left(\tilde{x}^{\prime}, \tilde{y}\right) \leq E u(\tilde{x}-\pi, \tilde{y})$ implies $E u\left(\tilde{x}^{\prime}-\right.$ $\Delta x, \tilde{y}) \leq E u(\tilde{x}-\Delta x-\pi, \tilde{y})$ where $\Delta x>0$ and $F_{\tilde{x}^{\prime}, \tilde{y}}$ is a CIMRI (CDMRI) of $F_{\tilde{x}, \tilde{y}}$

(ii) $u$ exhibits cross decreasing aversion to CIMRI (CDMRI) if $E u\left(\tilde{x}^{\prime}, \tilde{y}\right) \leq E u(\tilde{x}-\pi, \tilde{y})$ implies $E u\left(\tilde{x}^{\prime}, \tilde{y}-\Delta y\right) \leq E u(\tilde{x}-\pi, \tilde{y}-\Delta y)$ where $\Delta y>0$ and $F_{\tilde{x}^{\prime}, \tilde{y}}$ is a CIMRI (CDMRI) of $F_{\tilde{x}, \tilde{y}}$.

The next two results, which can be obtained as corollaries of Theorem 3, characterize these notions in terms of the underlying EU preferences and their behavioral implications.

Corollary 4 Assume $u_{11} \leq 0$ and $u_{12} \leq(\geq)$. The following are equivalent

(i) $u$ exhibits decreasing aversion to CIMRI (CDMRI).

(ii) There exists $\lambda$ such that for all $x$ and $y$, 


$$
u_{11}(x, y) \geq \lambda u_{1}(x, y), u_{111}(x, y) \geq \lambda u_{11}(x, y) \text { and } u_{112}(x, y) \geq(\leq) \lambda u_{12}(x, y)
$$

(iii) There exists $\Gamma(x, y ; \delta)$ and $\bar{\lambda}(\delta)>0$ such that, for $\delta>0, \Gamma_{1}(x, y ; \delta) \geq 0, \Gamma_{11}(x, y ; \delta) \geq 0$, $\Gamma_{12}(x, y ; \delta) \geq(\leq) 0$, and $u(x+\delta, y)=\bar{\lambda}(\delta) u(x, y)+\Gamma(x, y ; \delta)$

(iv) For all $\tilde{w}, \tilde{\epsilon}$, and $\tilde{y}$ such that $E[\tilde{\epsilon} \mid \tilde{y} \leq y, \tilde{w}=x] \leq(\geq) E[\tilde{\epsilon} \mid \tilde{w}=w]=0$ for all $w$ and $y, \alpha_{1}<\alpha_{2}$, $\Delta w>0, E u\left(\tilde{w}+\alpha_{2} \tilde{\epsilon}+\pi, \tilde{y}\right)=E u\left(\tilde{w}+\alpha_{1} \tilde{\epsilon}, \tilde{y}\right)$ and $E u\left(\tilde{w}+\Delta w+\alpha_{2} \tilde{\epsilon}+\hat{\pi}, \tilde{y}\right)=E u\left(\tilde{w}+\Delta w+\alpha_{1} \tilde{\epsilon}, \tilde{y}\right)$ imply that $\pi \geq \hat{\pi}$.

(v) If $\alpha^{*}(\tilde{w}, \tilde{x}, \tilde{y})$ maximizes $\operatorname{Eu}(\tilde{w}+\alpha \tilde{x}, \tilde{y})$, then $\alpha^{*}(\tilde{w}+\Delta w, \tilde{x}, \tilde{y}) \geq \alpha^{*}(\tilde{w}, \tilde{x}, \tilde{y})$ for all $\Delta w>0$ and $\tilde{w}, \tilde{x}$, and $\tilde{y}$ such that $E[\tilde{x} \mid \tilde{y} \leq y, \tilde{w}=w] \leq(\geq) E[\tilde{x} \mid \tilde{w}=w]=E[\tilde{x}]>0$ for all $w$ and $y$.

Corollary 5 Assume $u_{11} \leq 0$ and $u_{12} \leq(\geq)$. The following are equivalent

(i) $u$ exhibits cross decreasing aversion to CIMRI (CDMRI).

(ii) There exists $\lambda$ such that for all $x$ and $y$,

$$
u_{12}(x, y) \geq \lambda u_{1}(x, y), u_{112}(x, y) \geq \lambda u_{11}(x, y) \text { and } u_{122}(x, y) \geq(\leq) \lambda u_{12}(x, y)
$$

(iii) There exists $\Gamma(x, y ; \delta)$ and $\lambda(\delta)>0$ such that $\Gamma_{1}(x, y ; \delta) \geq 0, \Gamma_{11}(x, y ; \delta) \geq 0, \Gamma_{12}(x, y ; \delta) \geq$ $(\leq) 0$, and $u(x, y+\delta)=\lambda(\delta) u(x, y)+\Gamma(x, y ; \delta)$.

(iv) For all $\tilde{w}, \tilde{\epsilon}$, and $\tilde{y}$ such that $E[\tilde{\epsilon} \mid \tilde{y} \leq y, \tilde{w}=x] \leq(\geq) E[\tilde{\epsilon} \mid \tilde{w}=w]=0$ for all $w$ and $y, \alpha_{1}<\alpha_{2}$, $\Delta y>0, E u\left(\tilde{w}+\alpha_{2} \tilde{\epsilon}+\pi, \tilde{y}\right)=E u\left(\tilde{w}+\alpha_{1} \tilde{\epsilon}, \tilde{y}\right)$ and $E u\left(\tilde{w}+\alpha_{2} \tilde{\epsilon}+\hat{\pi}, \tilde{y}+\Delta y\right)=E u\left(\tilde{w}+\alpha_{1} \tilde{\epsilon}, \tilde{y}+\Delta y\right)$ imply that $\pi \geq \hat{\pi}$.

(v) If $\alpha^{*}(\tilde{w}, \tilde{x}, \tilde{y})$ maximizes $E u(\tilde{w}+\alpha \tilde{x}, \tilde{y})$, then $\alpha^{*}(\tilde{w}, \tilde{x}, \tilde{y}+\Delta y) \geq \alpha^{*}(\tilde{w}, \tilde{x}, \tilde{y})$ for all $\Delta y>0$ and $\tilde{w}, \tilde{x}$, and $\tilde{y}$ such that $E[\tilde{x} \mid \tilde{y} \leq y, \tilde{w}=w] \leq(\geq) E[\tilde{x} \mid \tilde{w}=w]=E[\tilde{x}]>0$ for all $w$ and $y$.

In the context of health insurance purchasing in the presence of uncertain health status (as the nonfinancial background risk), Corollaries 4 and 5 respectively establish the necessary and sufficient conditions for a deterministic improvement in wealth and in health to imply weaker aversion to CIMRI and reduced health insurance coverage if there are proportional premium loadings and, conditional on his initial wealth, an individual's health care cost is non-positive expectation dependent on his health status. 


\section{Conclusion}

This paper defines and characterizes bivariate stochastic deteriorations "correlation-increasing marginal risk increase" (CIMRI) and "correlation-decreasing marginal risk increase" (CDMRI). We show that a CIMRI (CDMRI) is what results from taking a zero-mean financial risk in the presence of a nonfinancial risk that is correlated with the financial risk in the sense of positive (negative) expectation dependence and is the stochastic deterioration disliked by all EU maximizers who are both correlationaverse (-loving) and marginal risk averse. Moreover, a CIMRI (CDMRI) has a necessary and sufficient "integral condition" that is a generalization of that for a "mean-preserving increase in risk" obtained in Rothschild and Stiglitz (1970) and implies and is implied by a sense in which a CIMRI (CDMRI) can be decomposed into a correlation increase (decrease) and a marginal risk increase. Together with a comprehensive characterization of attitudes towards financial risk-taking in the presence of a correlated non-financial background risk that we also offer, these results in turn provides an intuitive explanation for, as well as a significant generalization of, existing results on optimal choice of endogenous risk in the presence of financial and non-financial background risks. We further characterize a measure of aversion to CIMRI (CDMRI) and demonstrate their behavioral implications in the contexts of risk management decisions.

\section{Appendix}

\section{Proof of Theorem 1.}

$[(\mathrm{i}) \Rightarrow(\mathrm{ii})]$

Since letting $u^{+}(x, y)=x$ and $u^{-}(x, y)=-x$, we have $u_{11}^{+} \leq 0, u_{12}^{+} \leq 0, u_{11}^{-} \leq 0$ and $u_{12}^{-} \leq 0$,

$$
E u^{+}\left(\tilde{x}^{\prime}, \tilde{y}\right)-E u^{+}(\tilde{x}, \tilde{y})=\int_{-a}^{a} t d[G(t, a)-F(t, a)]=-\int_{-a}^{a}[G(t, a)-F(t, a)] d t \leq 0
$$

and

$$
E u^{-}\left(\tilde{x}^{\prime}, \tilde{y}\right)-E u^{-}(\tilde{x}, \tilde{y})=-\int_{-a}^{a} t d[G(t, a)-F(t, a)]=\int_{-a}^{a}[G(t, a)-F(t, a)] d t \leq 0
$$

imply $\int_{-a}^{a}[G(t, a)-F(t, a)] d t=0$.

To show $\left[\int_{-a}^{a} \int_{-a}^{z(y)}[G(x \mid \tilde{y}=y)-F(x \mid \tilde{y}=y)] d x d F(a, y) \geq 0\right.$ for all $z(y)$ that is non-increasing in $y]$ is necessary for $\left[E u(\tilde{x}, \tilde{y}) \geq E u\left(\tilde{x}^{\prime}, \tilde{y}\right)\right.$ for all $u_{11} \leq 0$ and $\left.u_{12} \leq 0\right]$, suppose there exists $\hat{z}(y)$ non-increasing in $y$ such that $\int_{-a}^{a} \int_{-a}^{\hat{z}(y)}[G(x \mid \tilde{y}=y)-F(x \mid \tilde{y}=y)] d x d F(a, y)<0$. Letting

$$
\hat{u}(x, y)=-\max \{\hat{z}(y)-x, 0\}
$$




$$
\begin{aligned}
& E \hat{u}\left(\tilde{x}^{\prime}, \tilde{y}\right)-E \hat{u}(\tilde{x}, \tilde{y})=\int_{-a}^{b} \int_{-a}^{b} \hat{u}(x, y) d[G(x, y)-F(x, y)] \\
= & \int_{-a}^{a} \int_{-a}^{a} \hat{u}(x, y) d[G(x \mid \tilde{y}=y)-F(x \mid \tilde{y}=y)] d F(a, y) \\
= & -\int_{-a}^{a} \int_{-a}^{\hat{z}(y)}[G(x \mid \tilde{y}=y)-F(x \mid \tilde{y}=y)] d x d F(a, y)>0 .
\end{aligned}
$$

By an argument in Fishburn and Vickson $(1978$, p.76), it is possible to approximate $\hat{u}(x, y)$ by a twicedifferentiable function $u(x, y)$ such that $u_{11} \leq 0$ and $u_{12} \leq 0$ but $E u\left(\tilde{x}^{\prime}, \tilde{y}\right)-E u(\tilde{x}, \tilde{y})>0$.

Given what is shown above, the necessity of $\left[\int_{-a}^{a} \int_{z(y)}^{a}[G(x \mid \tilde{y}=y)-F(x \mid \tilde{y}=y)] d x d F(a, y) \leq 0\right.$ for all $z(y)$ that is non-decreasing in $y]$ for $\left[E u(\tilde{x}, \tilde{y}) \geq E u\left(\tilde{x}^{\prime}, \tilde{y}\right)\right.$ for all $u_{11} \leq 0$ and $\left.u_{12} \geq 0\right]$ follows from the following observations. First, $E u(\tilde{x}, \tilde{y}) \geq E u\left(\tilde{x}^{\prime}, \tilde{y}\right)$ for all $u_{11} \leq 0$ and $u_{12} \geq 0$ if and only if $E u(-\tilde{x}, \tilde{y}) \geq E u\left(-\tilde{x}^{\prime}, \tilde{y}\right)$ for all $u_{11} \leq 0$ and $u_{12} \leq 0$. Second, $F(x, y)$ and $G(x, y)$ are the distribution functions of $(\tilde{x}, \tilde{y})$ and $\left(\tilde{x}^{\prime}, \tilde{y}\right)$ and $\int_{-a}^{a} \int_{-a}^{z(y)}[G(x \mid \tilde{y}=y)-F(x \mid \tilde{y}=y)] d x d F(a, y) \geq 0$ for all $z(y)$ that is non-increasing in $y$ if and only if $\hat{F}(x, y)$ and $\hat{G}(x, y)$ are the distribution functions of $(-\tilde{x}, \tilde{y})$ and $\left(-\tilde{x}^{\prime}, \tilde{y}\right)$ and $\int_{-a}^{a} \int_{z(y)}^{a}[\hat{G}(x \mid \tilde{y}=y)-\hat{F}(x \mid \tilde{y}=y)] d x d \hat{F}(a, y) \leq 0$ for all $z(y)$ that is non-decreasing in $y$.

$[(i i) \Rightarrow($ iii $)]$ In proving this result, we adopt the following notation: Given a function $H(x):[-a, a] \rightarrow R$,

$$
H\left(x^{-}\right) \equiv \lim _{\delta \uparrow 0} H(x+\delta) \quad \text { and } \quad H\left(x^{+}\right) \equiv \lim _{\delta \downarrow 0} H(x+\delta) .
$$

Furthermore, if $H(x, y):[-a, a]^{2} \rightarrow R$ is not continuous in $y$, denoting by $D_{H} \equiv\left\{\hat{y}_{1}, \hat{y}_{2}, \ldots\right\}$ the set of its discontinuity points and $I_{+}$the set of non-negative integers and setting $\hat{y}_{0}=-a$, we define

$$
\begin{gathered}
\int_{-a}^{y}\left[H(x, s)-H\left(x, s^{-}\right)\right] \\
\equiv \int_{-a}^{y} H_{y}(x, s) d s+\sum_{i=1}^{\max \left\{i \in I_{+}: \hat{y}_{i} \leq y\right\}}\left[H\left(x, \hat{y}_{i}\right)-H\left(x, \hat{y}_{i}^{-}\right)\right]+\sum_{i=1}^{\max \left\{i \in I_{+}: \hat{y}_{i}<y\right\}}\left[H\left(x, \hat{y}_{i}^{+}\right)-H\left(x, \hat{y}_{i}\right)\right]
\end{gathered}
$$

13 where

$$
\begin{gathered}
H_{y}(x, y) \equiv \begin{cases}\frac{H(x, y)-H\left(x, y^{-}\right)}{y-y^{-}} & \text {for } y \in[-a, a] \backslash D_{H} ; \\
0 & \text { for } y \in D_{H}\end{cases} \\
\sum_{i=1}^{0}\left[H\left(x, \hat{y}_{i}\right)-H\left(x, \hat{y}_{i}^{-}\right)\right]=\sum_{i=1}^{0}\left[H\left(x, \hat{y}_{i}^{+}\right)-H\left(x, \hat{y}_{i}\right)\right] \equiv 0 .
\end{gathered}
$$

(Thus in the case where $H(x, y)$ is continuous in $y$, i.e., $D_{H}$ is empty, $\int_{-a}^{y}\left[H(x, s)-H\left(x, s^{-}\right)\right]=$

\footnotetext{
${ }^{13}$ Note that the last term vanishes if $H(x, y)$ is right-continuous in $y$.
} 
$\left.\int_{-a}^{y} H_{y}(x, s) d s.\right)$ Then we clearly always have $H(x, y)=\int_{-a}^{y}\left[H(x, s)-H\left(x, s^{-}\right)\right]+H(x,-a)$.

Now consider the function $\Phi(x, y):[-a, a]^{2} \rightarrow R^{2}:$

$$
\Phi(x,-a) \equiv G(x,-a)-F(x,-a)
$$

and, for $y>-a$,

$$
\Phi(x, y) \equiv G(x, y)-G\left(x, y^{-}\right)-\left[F(x, y)-F\left(x, y^{-}\right)\right]+\Psi\left(x, y^{-}\right) .
$$

where the function $\Psi(x, y):[-a, a]^{2} \rightarrow R^{2}$ is defined as follows. Let

$$
Z_{\Phi} \equiv\{y \in[-a, a]: \Phi(x, y)=0 \text { for all } x\}
$$

$\Psi(x, y)=\Phi(x, y)$ for $y \in Z_{\Phi}$. For each $y \in[-a, a] \backslash Z_{\Phi}$, let $m(y) \in\{0,1,2, \ldots\}$ be the number of times $\Phi(x, y)$ crosses the $x$-axis and $x_{1}^{y}, x_{2}^{y}, \ldots$ the "crossings" : $x_{0}^{y} \equiv-a, x_{m(y)+1}^{y} \equiv a$, for $m(y) \in\{1,2, \ldots\}$, and $k=0,1, \ldots,[m(y)-1], \Phi\left(x_{m(y)-k}^{y}, y\right)=0, \int_{x_{m(y)-k}^{y}}^{x_{m(y)-k+1}^{y}} \Phi(x, y) d x \neq 0$, and if $\Phi(x, y) \geq(\leq) 0$ for $x \in\left[x_{m(y)-k}^{y}, x_{m(y)-k+1}^{y}\right]$, then $\Phi(x, y) \leq(\geq) 0$ for $x \in\left[x_{m(y)-k-1}^{y}, x_{m(y)-k}^{y}\right]$. For each $y \in$ $[-a, a] \backslash Z_{\Phi}, m(y) \in\{0,1,2, \ldots\}$ and $k=0,1, \ldots, m(y)$, define

$$
\begin{gathered}
A_{m(y)-k}^{y} \equiv \int_{x_{m(y)-k}^{y}}^{x_{m(y)-k+1}^{y}} \Phi(x, y) d x \\
\alpha_{m(y)-k}^{y}=\max \left\{0, \frac{A_{m(y)-k}^{y}+\sum_{j=1}^{k}\left(1-\alpha_{m(y)-k+j}^{y}\right) A_{m(y)-k+j}^{y}}{\left|A_{m(y)-k}^{y}\right|}\right\}
\end{gathered}
$$

(where for expositional ease we define $\left.\sum_{j=1}^{0}\left(1-\alpha_{m(y)+j}^{y}\right) A_{m(y)+j}^{y} \equiv 0\right)$ and

$$
\Psi(x, y) \equiv \begin{cases}\alpha_{m(y)}^{y} \Phi(x, y) & \text { for } x \in\left[x_{m(y)}^{y}, a\right] \\ \cdots \ldots & \\ \alpha_{m(y)-k}^{y} \Phi(x, y) & \text { for } x \in\left[x_{m(y)-k}^{y}, x_{m(y)-k+1}^{y}\right] \\ \cdots \cdots & \\ \alpha_{0}^{i} \Phi(x, y) & \text { for } x \in\left[-a, x_{1}^{y}\right] .\end{cases}
$$

We immediately have $\sum_{j=0}^{k}\left(1-\alpha_{m(y)-k+j}^{y}\right) A_{m(y)-k+j}^{y} \leq 0$ for $m(y) \in\{0,1,2, \ldots\}$ and $k=0,1, \ldots, m(y)$, 
14 which in turn implies that $\alpha_{m(y)-k}^{y}>0$ if and only if $A_{m(y)-k}^{y}+\sum_{j=1}^{k}\left(1-\alpha_{m(y)-k+j}^{y}\right) A_{m(y)-k+j}^{y}>0$ and $A_{m(y)-k}^{y}>0$ (or equivalently $\Phi(x, y) \geq 0$ for $x \in\left[x_{m(y)-k}^{y}, x_{m(y)-k+1}^{y}\right]$ ). We are to further show the following.

(a) $\int_{-a}^{x} \Phi(t, y) d t \geq 0$ for all $x$ and $y$;

(b) $\int_{-a}^{a} \Phi(t, a) d t=0$;

(c) $\Psi(x, y) \geq 0$ for all $x$ and $y$;

(d) $\Psi(a, y)=0$ for all $y$;

(e) $\Psi(x, a)=0$ for all $x$;

(f) $\int_{-a}^{a}[\Phi(t, y)-\Psi(t, y)] d t=0$ for all $y$;

(g) $\int_{-a}^{x}[\Phi(t, y)-\Psi(t, y)] d t \geq 0$ for all $x$ and $y$.

(a)

Let $\tau(x, y)$ be such that, for $y \in Z_{\Phi}, \tau(x, y) \equiv a$ and, for $y \in[-a, a] \backslash Z_{\Phi}, m(y) \in\{0,1,2, \ldots\}$ and $k=0,1, \ldots, m(y)$, letting $\check{x}_{m(y)-k}^{y} \in\left[x_{m(y)-k}^{y}, x_{m(y)-k+1}^{y}\right]$ be such that $\int_{x_{m(y)-k}^{y}}^{\check{x}_{y(y)-k}^{y}} \Phi(x, y) d x=$ $\int_{x_{m(y)-k}^{y}}^{x_{m(y)-k+1}^{y}} \alpha_{m(y)-k} \Phi(x, y) d x$

$$
\tau(x, y) \equiv \begin{cases}x & \text { for } x \in\left[-a, \check{x}_{0}^{y}\right) ; \\ x_{1}^{y} & \text { for } x \in\left[\check{x}_{0}^{y}, x_{1}^{y}\right) ; \\ \ldots \ldots & \text { for } x \in\left[x_{m(y)-k}^{y}, \check{x}_{m(y)-k}^{i}\right) ; \\ x_{m(y)-k+1}^{y} & \text { for } x \in\left[\check{x}_{m(y)-k}^{y}, x_{m(y)-k+1}^{y}\right) ; \\ \ldots \ldots & \text { for } x \in\left[x_{m(y)}^{y}, \check{x}_{m(y)}^{y}\right) ; \\ x & \text { for } x \in\left[\check{x}_{m(y)}^{y}, a\right] .\end{cases}
$$

Then clearly $\int_{-a}^{x_{m(y)-k+1}^{y}} \Psi(t, y) d t=\int_{-a}^{\tau\left(x_{m(y)-k+1}^{y}, y\right)} \Phi(t, y) d t$. Furthermore $\tau(x, y) \geq x$ for all $x$ and $y$ and $\tau(a, y)=a$ for all $y$. Let $\Psi^{*}(x, y)$ be such that, for all $x$ and $y$,

$$
\int_{-a}^{x} \Psi^{*}(t, y) d t=\int_{-a}^{\tau(x, y)} \Phi(t, y) d t
$$

\footnotetext{
${ }^{14}$ If $A_{m(y)-k}^{y}+\sum_{j=1}^{k}\left(1-\alpha_{m(y)-k+j}^{y}\right) A_{m(y)-k+j}^{y} \leq 0$, clearly $\alpha_{m(y)}^{y}=0$ and thus $\sum_{j=0}^{k}\left(1-\alpha_{m(y)-k+j}^{y}\right) A_{m(y)-k+j}^{y}=$ $A_{m(y)-k}^{y}+\sum_{j=1}^{k}\left(1-\alpha_{m(y)-k+j}^{y}\right) A_{m(y)-k+j}^{y} \leq 0$. If $A_{m(y)-k}^{y}+\sum_{j=1}^{k}\left(1-\alpha_{m(y)-k+j}^{y}\right) A_{m(y)-k+j}^{y}>0$, it is easy to see that in the case of $k=0, \alpha_{m(y)}^{y}=1$ and thus $\left(1-\alpha_{m(y)}^{y}\right) A_{m(y)}^{y}=0$ and if $\sum_{j=0}^{k}\left(1-\alpha_{m(y)-k+j}^{y}\right) A_{m(y)-k+j}^{y} \leq 0$, then $\sum_{j=0}^{k+1}\left(1-\alpha_{m(y)-k+j}^{y}\right) A_{m(y)-k+j}^{y} \leq 0$ and thus by induction $\sum_{j=0}^{k}\left(1-\alpha_{m(y)-k+j}^{y}\right) A_{m(y)-k+j}^{y} \leq 0$ for all $k=$ $0,1, \ldots, m(y)$.
} 
and define

$$
\Phi^{*}(x, y) \equiv G(x, y)-G\left(x, y^{-}\right)-\left[F(x, y)-F\left(x, y^{-}\right)\right]+\Psi^{*}\left(x, y^{-}\right) .
$$

Then clearly $\int_{-a}^{x_{m(y)-k+1}^{y}} \Phi(t, y) d t=\int_{-a}^{x_{m(y)-k+1}^{y}} \Phi^{*}(t, y) d t$ and, for any $x$ and $y$,

$$
\begin{aligned}
& \int_{-a}^{x} \Phi^{*}(t, y) d t \\
= & \int_{-a}^{x}\left[G(t, y)-G\left(t, y^{-}\right)-F(t, y)+F\left(t, y^{-}\right)\right] d t+\int_{-a}^{x} \Psi^{*}\left(t, y^{-}\right) d t \\
= & \int_{-a}^{x}\left[G(t, y)-G\left(t, y^{-}\right)-F(t, y)+F\left(t, y^{-}\right)\right] d t+\int_{-a}^{\tau\left(x, y^{-}\right)} \Phi^{*}\left(t, y^{-}\right) d t .
\end{aligned}
$$

For a $\left(x^{*}, y^{*}\right) \in[-a, a]^{2}$, let $z^{*}(y)$ be such that $z^{*}(y)=-a$ for $y>y^{*}, z^{*}\left(y^{*}\right)=x^{*}$ and $z^{*}\left(y^{-}\right)=$ $\tau\left(z^{*}(y), y^{-}\right)$for $y \leq y^{*}$. Then given $\tau(x, y) \geq x, z^{*}(y)$ is clearly non-increasing and, for $y \leq y^{*}$,

$\int_{-a}^{z^{*}(y)} \Phi^{*}(t, y) d t$

$=\int_{-a}^{z^{*}(y)}\left[G(t, y)-G\left(t, y^{-}\right)-F(t, y)+F\left(t, y^{-}\right)\right] d t+\int_{-a}^{\tau\left(z^{*}(y), y^{-}\right)} \Phi^{*}\left(t, y^{-}\right) d t$,

$=\int_{-a}^{z^{*}(y)}\left[G(t, y)-G\left(t, y^{-}\right)-F(t, y)+F\left(t, y^{-}\right)\right] d t+\int_{-a}^{z^{*}\left(y^{-}\right)} \Phi^{*}\left(t, y^{-}\right) d t$,

or equivalently,

$$
\int_{-a}^{z^{*}(y)} \Phi^{*}(t, y) d t-\int_{-a}^{z^{*}\left(y^{-}\right)} \Phi^{*}\left(t, y^{-}\right) d t=\int_{-a}^{z^{*}(y)}\left[G(t, y)-G\left(t, y^{-}\right)-F(t, y)+F\left(t, y^{-}\right)\right] d t .
$$

Thus, noting that

$$
G(x \mid \tilde{y}=y)=\frac{G(x, y)-G\left(x, y^{-}\right)}{G(a, y)-G\left(a, y^{-}\right)} \text {and } F(x \mid \tilde{y}=y)=\frac{F(x, y)-F\left(x, y^{-}\right)}{F(a, y)-F\left(a, y^{-}\right)}
$$

$$
\begin{aligned}
& \int_{-a}^{x^{*}} \Phi^{*}\left(t, y^{*}\right) d t \\
= & \int_{-a}^{z^{*}\left(y^{*}\right)} \Phi^{*}\left(t, y^{*}\right) d t \\
= & \int_{-a}^{y^{*}}\left[\int_{-a}^{z^{*}(y)} \Phi^{*}(t, y) d t-\int_{-a}^{z^{*}\left(y^{-}\right)} \Phi^{*}\left(t, y^{-}\right) d t\right] \\
= & \int_{-a}^{a}\left\{\int_{-a}^{z^{*}(y)}\left[G(t, y)-G\left(t, y^{-}\right)-F(t, y)+F\left(t, y^{-}\right)\right] d t\right\} \\
= & \int_{-a}^{a} \int_{-a}^{z^{*}(y)}[G(t \mid \tilde{y}=y)-F(t \mid \tilde{y}=y)] d t d F(a, y) \geq 0
\end{aligned}
$$

where the inequality is implied by the hypothesis that $\int_{-a}^{a} \int_{-a}^{z(y)}[G(x \mid \tilde{y}=y)-F(x \mid \tilde{y}=y)] d x d F(a, y) \geq$ 0 for all $z(y)$ that is non-increasing in $y$. That is, we have $\int_{-a}^{x} \Phi^{*}(t, y) d t \geq 0$ for all $x$ and $y$. Then clearly $\int_{-a}^{x_{m(y)-k+1}^{y}} \Phi(t, y) d t=\int_{-a}^{x_{m(y)-k+1}^{y}} \Phi^{*}(t, y) d t \geq 0$ for $m(y) \in\{0,1,2, \ldots\}$ and $k=0,1, \ldots, m(y)$, which implies $\int_{-a}^{x} \Phi(t, y) d t \geq 0$ for all $x$ and $y$ : If $x \in\left[x_{m(y)-k}^{y}, x_{m(y)-k+1}^{y}\right]$ such that $\Phi(x, y) \leq 0$ for all $x \in\left[x_{m(y)-k}^{y}, x_{m(y)-k+1}^{y}\right]$, then

$$
\int_{-a}^{x} \Phi(t, y) d t \geq \int_{-a}^{x} \Phi(t, y)+\int_{x}^{x_{m(y)-k+1}^{y}} \Phi(t, y) d t=\int_{-a}^{x_{m(y)-k+1}^{y}} \Phi(t, y) d t \geq 0 .
$$

If $x \in\left[x_{m(y)-k}^{y}, x_{m(y)-k+1}^{y}\right]$ such that $\Phi(x, y) \geq 0$ for all $x \in\left[x_{m(y)-k}^{y}, x_{m(y)-k+1}^{y}\right]$, then

$$
\int_{-a}^{x} \Phi(t, y) d t=\int_{-a}^{x_{m(y)-k}^{y}} \Phi(t, y)+\int_{x_{m(y)-k}^{y}}^{x} \Phi(t, y) d t \geq \int_{-a}^{x_{m(y)-k}^{y}} \Phi(t, y) d t \geq 0 .
$$


(b)

$$
\begin{aligned}
& \text { Since } \tau(a, y)=a \text { for all } y, \\
& \int_{-a}^{a} \Phi(t, y) d t \\
= & \int_{-a}^{a}\left[G(t, y)-G\left(t, y^{-}\right)-F(t, y)+F\left(t, y^{-}\right)\right] d t+\int_{-a}^{a} \Psi\left(t, y^{-}\right) \\
= & \int_{-a}^{a}\left[G(t, y)-G\left(t, y^{-}\right)-F(t, y)+F\left(t, y^{-}\right)\right] d t+\int_{-a}^{a} \Phi\left(t, y^{-}\right) .
\end{aligned}
$$

Thus

$$
\begin{aligned}
& \int_{-a}^{a} \Phi\left(t, y^{*}\right) d t \\
&= \int_{-a}^{y^{*}}\left[\int_{-a}^{a} \Phi(t, y) d t-\int_{-a}^{a} \Phi\left(t, y^{-}\right)\right] \\
&=\left.\int_{-a}^{y^{*}}\left\{\int_{-a}^{a} G(t, y)-G\left(t, y^{-}\right)-F(t, y)+F\left(t, y^{-}\right)\right] d t\right\} \\
&= \int_{-a}^{a}\left[G\left(t, y^{*}\right)-F\left(t, y^{*}\right)\right] d t . \\
& \int_{-a}^{a}[G(t, a)-F(t, a)] d t=0 \text { then implies } \int_{-a}^{a} \Phi(t, a) d t=0 .
\end{aligned}
$$

(c)

$\Psi(x, y) \geq 0$ for all $x$ and $y$ since $\alpha_{m(y)-k}^{y} \geq 0$ for all $k=0,1, \ldots, m(y)$ and $\alpha_{m(y)-k}^{y}>0$ only if $\Phi(x, y) \geq 0$ for $x \in\left[x_{m(y)-k}^{y}, x_{m(y)-k+1}^{y}\right]$.

Given the definition of $\Psi(x, y), \Phi(a, y)=0$ for all $y$ implies $\Psi(a, y)=0$ for all $y$.

(e)

$\Psi(x, a)=0$ for all $x$ since $\int_{-a}^{x} \Phi(t, a) d t \geq 0$ for all $x$ and $\int_{-a}^{a} \Phi(t, a) d t=0$ imply $\alpha_{m(a)-k}^{a}=0$ for $k=0,1, \ldots, m(a):$ If the set $\left\{k \in\{0,1, \ldots, m(a)\}: \alpha_{m(a)-k}^{a}>0\right\}$ is non-empty, letting $\underline{k} \equiv \min \{k:$ $\left.\alpha_{m(a)-k}^{a}>0\right\}, A_{m(a)-\underline{k}}^{a}+\sum_{j=1}^{\underline{k}}\left(1-\alpha_{m(a)-\underline{k}+j}^{a}\right) A_{m(a)-\underline{k}+j}^{a}=\int_{x_{m(a)-\underline{k}}^{y}}^{a} \Phi(x, a) d x>0$, which (given $\left.\int_{-a}^{a} \Phi(t, a) d t=0\right)$ implies $\int_{-a}^{x_{m(a)-\underline{k}}^{y}} \Phi(x, a) d x<0$.

If $\alpha_{m(y)-k}^{y}=0$ for $k=0,1, \ldots, m(y)$, then $\int_{-a}^{a}[\Phi(t, y)-\Psi(t, y)] d t=\int_{-a}^{a} \Phi(t, y) d t \geq 0$ (by (a)). But we also know $\int_{-a}^{a}[\Phi(t, y)-\Psi(t, y)] d t=\sum_{j=0}^{m(y)}\left(1-\alpha_{j}^{y}\right) A_{j}^{y} \leq 0$. Hence we must have $\int_{-a}^{a}[\Phi(t, y)-\Psi(t, y)] d t=0$.

If the set $\left\{k \in\{0,1, \ldots, m(a)\}: \alpha_{m(y)-k}^{y}>0\right\}$ is non-empty, let $\bar{k} \equiv \max \{k \in\{0,1, \ldots, m(a)\}$ : $\left.\alpha_{m(y)-k}^{y}>0\right\}$. Then $\int_{x_{m(y)-\bar{k}}^{y}}^{a}[\Phi(t, y)-\Psi(t, y)] d t=\sum_{j=m(y)-\bar{k}}^{m(y)}\left(1-\alpha_{j}^{y}\right) A_{j}^{y}=0$. In the case of $\bar{k}=$ $m(y)$, we immediately have $\int_{-a}^{a}[\Phi(t, y)-\Psi(t, y)] d t=0$. If $\bar{k}<m(y), \alpha_{j}^{y}=0$ for $j=0,1, \ldots,[m(y)-\bar{k}]$ implies $\int_{-a}^{x_{m(y)-\bar{k}}}[\Phi(t, y)-\Psi(t, y)] d t=\int_{-a}^{x_{m(y)-\bar{k}}} \Phi(t, y) d t \geq 0$ (by (a)). But given

$$
A_{0}^{y}+\sum_{j=1}^{m(y)}\left(1-\alpha_{j}^{y}\right) A_{j}^{y}
$$


$\geq \int_{-a}^{a}[\Phi(t, y)-\Psi(t, y)] d t$

$=\int_{-a}^{x_{m(y)-\bar{k}}}[\Phi(t, y)-\Psi(t, y)] d t+\int_{x_{m(y)-\bar{k}}^{y}}^{a}[\Phi(t, y)-\Psi(t, y)] d t$

$=\int_{-a}^{x_{m(y)-\bar{k}}}[\Phi(t, y)-\Psi(t, y)] d t$

(where the inequality holds because (a) implies $A_{0}^{y} \geq 0$ ), $\int_{-a}^{x_{m(y)-\bar{k}}}[\Phi(t, y)-\Psi(t, y)] d t>0$ implies $\alpha_{0}^{y}>0$, which is a contradiction. We thus have $\int_{-a}^{x_{m(y)-\bar{k}}} \Phi(t, y) d t=0$ and

$$
\int_{-a}^{a}[\Phi(t, y)-\Psi(t, y)] d t=\int_{-a}^{x_{m(y)-\bar{k}}} \Phi(t, y) d t+\int_{x_{m(y)-\bar{k}}^{y}}^{a}[\Phi(t, y)-\Psi(t, y)] d t=0 .
$$

(g)

$$
\int_{x_{m(y)-k}^{y}}^{a}[\Phi(t, y)-\Psi(t, y)] d t=\sum_{j=0}^{k}\left(1-\alpha_{m(y)-k+j}^{y}\right) A_{m(y)-k+j}^{i} \leq 0 \text { for } k=0,1, \ldots, m(y) \text { implies }
$$

$\int_{x}^{a}[\Phi(t, y)-\Psi(t, y)] d t \leq 0$ for all $x \in[-a, a]:$ If $x \in\left[x_{m(y)-k}^{y}, x_{m(y)-k+1}^{y}\right]$ such that $\Phi(x, y)-\Psi(x, y) \leq$ 0 for all $x \in\left[x_{m(y)-k}^{y}, x_{m(y)-k+1}^{y}\right]$, then

$$
\begin{aligned}
& \int_{x}^{a}[\Phi(t, y)-\Psi(t, y)] d t \\
= & \int_{x}^{x_{m(y)-k+1}^{y}}[\Phi(t, y)-\Psi(t, y)] d t+\int_{x_{m(y)-k+1}^{y}}^{a}[\Phi(t, y)-\Psi(t, y)] d t \leq \int_{x_{m(y)-k+1}^{y}}^{a}[\Phi(t, y)-\Psi(t, y)] d t \leq 0 .
\end{aligned}
$$

If $x \in\left[x_{m(y)-k}^{y}, x_{m(y)-k+1}^{y}\right]$ such that $\Phi(x, y)-\Psi(x, y) \geq 0$ for all $x \in\left[x_{m(y)-k}^{y}, x_{m(y)-k+1}^{y}\right]$, then

$\int_{x}^{a}[\Phi(t, y)-\Psi(t, y)] d t$

$\leq \int_{x_{m(y)-k}^{y}}^{x}[\Phi(t, y)-\Psi(t, y)] d t+\int_{x}^{a}[\Phi(t, y)-\Psi(t, y)] d t \leq \int_{x_{m(y)-k}^{y}}^{a}[\Phi(t, y)-\Psi(t, y)] d t \leq 0$.

Given $\int_{-a}^{a}[\Phi(t, y)-\Psi(t, y)] d t=0$, we thus have $\int_{-a}^{x}[\Phi(t, y)-\Psi(t, y)] d t \geq 0$.

Now define

$$
H(x, y)=F(x, y)+\Psi(x, y) \text { and } H(x \mid \tilde{y}=y) \equiv \frac{H(x, y)-H\left(x, y^{-}\right)}{H(a, y)-H\left(a, y^{-}\right)}
$$

We clearly have $H(x, y) \geq F(x, y), H(x, a)=F(x, a), H(a, y)=F(a, y)$. And since

$$
\begin{aligned}
& G(x, y)-G\left(x, y^{-}\right)-\left[H(x, y)-H\left(x, y^{-}\right)\right] \\
&= F(x, y)-F\left(x, y^{-}\right)+\Phi(x, y)-\Psi\left(x, y^{-}\right)-\left[F(x, y)+\Psi(x, y)-F\left(x, y^{-}\right)-\Psi\left(x, y^{-}\right)\right] \\
&= \Phi(x, y)-\Psi(x, y) \\
&\text { and (given } H(a, y)=F(a, y)=G(a, y) \text { for all } y)
\end{aligned}
$$

$$
G(x \mid \tilde{y}=y)-H(x \mid \tilde{y}=y)=\frac{G(x, y)-G\left(x, y^{-}\right)-\left[H(x, y)-H\left(x, y^{-}\right)\right]}{G(a, y)-G\left(a, y^{-}\right)},
$$

we have $\int_{-a}^{x}[G(t \mid \tilde{y}=y)-H(t \mid \tilde{y}=y)] d t \geq 0$ and $\int_{-a}^{a}[G(t \mid \tilde{y}=y)-H(t \mid \tilde{y}=y)] d t=0$.

Note that $H(x, y)$ may or may not be a distribution function as $H(x \mid \tilde{y}=y)$ may be decreasing. 
Define

$$
\Delta(x \mid y) \equiv \begin{cases}-H(x \mid \tilde{y}=y)+H\left(x^{-} \mid \tilde{y}=y\right) & \text { if } H(x \mid \tilde{y}=y)-H\left(x^{-} \mid \tilde{y}=y\right)<0 \\ 0 & \text { or } h(x \mid y)<0 \text { where } \int_{-a}^{x} h(t \mid y) d t=H(x \mid \tilde{y}=y) \\ \text { otherwise }\end{cases}
$$

and

$$
\begin{gathered}
\hat{F}(x, y) \equiv \frac{F(x, y)+\int_{-a}^{y}\left[\int_{-a}^{x} \Delta(t \mid s)\right] d H(a, s)}{F(a, a)+\int_{-a}^{a}\left[\int_{-a}^{a} \Delta(t \mid s)\right] d H(a, s)}, \hat{G}(x, y) \equiv \frac{G(x, y)+\int_{-a}^{y}\left[\int_{-a}^{x} \Delta(t \mid s)\right] d H(a, s)}{G(a, a)+\int_{-a}^{a}\left[\int_{-a}^{a} \Delta(t \mid s)\right] d H(a, s)}, \\
\hat{H}(x, y) \equiv \frac{H(x, y)+\int_{-a}^{y}\left[\int_{-a}^{x} \Delta(t \mid s)\right] d H(a, s)}{H(a, a)+\int_{-a}^{a}\left[\int_{-a}^{a} \Delta(t \mid s)\right] d H(a, s)}
\end{gathered}
$$

where $F(a, a)=G(a, a)=H(a, a)=1($ since $H(x, a)=F(x, a)$ and $H(a, y)=F(a, y)) . \hat{F}(x, y)$, $\hat{G}(x, y)$ and $\hat{H}(x, y)$ are thus all distribution functions. Letting

$$
\gamma \equiv \frac{1}{1+\int_{-a}^{a}\left[\int_{-a}^{a} \Delta(t \mid s)\right] d H(a, s)}
$$

clearly $\hat{G}(x, y)-\hat{F}(x, y)=\gamma[G(x, y)-F(x, y)]$ for all $x$ and $y$. Thus for all $x$ and $y, \hat{H}(x, y)-\hat{F}(x, y)=$ $\gamma[H(x, y)-F(x, y)] \geq 0 \hat{H}(x, a)-\hat{F}(x, a)=\gamma[H(x, a)-F(x, a)]=0, \int_{-a}^{x}[\hat{G}(t \mid \tilde{y}=y)-\hat{H}(t \mid \tilde{y}=$ $y)] d t=\gamma \int_{-a}^{x}[G(t \mid \tilde{y}=y)-H(t \mid \tilde{y}=y)] d t \geq 0$ and $\int_{-a}^{a}[\hat{G}(t \mid \tilde{y}=y)-\hat{H}(t \mid \tilde{y}=y)] d t=\gamma \int_{-a}^{a}[G(t \mid \tilde{y}=$ $y)-H(t \mid \tilde{y}=y)] d t=0$.

$[($ iii $) \Rightarrow(\mathrm{i})]$

$$
\begin{aligned}
& E u\left(\tilde{x}^{\prime}, \tilde{y}\right)-E u(\tilde{x}, \tilde{y})=\int_{-a}^{b} \int_{-a}^{b} u(x, y) d[G(x, y)-F(x, y)] \\
= & \frac{1}{\gamma} \int_{-a}^{b} \int_{-a}^{b} u(x, y) d[\hat{G}(x, y)-\hat{F}(x, y)] \\
= & \frac{1}{\gamma}\left\{\int_{-a}^{b} \int_{-a}^{b} u(x, y) d[\hat{G}(x, y)-\hat{H}(x, y)]+\int_{-a}^{b} \int_{-a}^{b} u(x, y) d[\hat{H}(x, y)-\hat{F}(x, y)]\right\}
\end{aligned}
$$

which is non-positive since, given $\hat{G}(x, y)$ being a $\mathrm{Cl}(\mathrm{CD})$ of $\hat{H}(x, y)$ and $\hat{H}(x, y)$ an MRI of $\hat{F}(x, y)$, by Lemmas 3 and 4 , both terms are non-positive for all $u$ such that $u_{11} \leq 0$ and $u_{12} \leq(\geq) 0$.

$[(i) \Longleftrightarrow$ (iv)]. The proof is completely analogous to Theorem 3.4 of Muller and Scarsini (2012) with $\mathcal{F}$ being the class of CIMRI (CDMRI) averse $u(x, y)$ and $M$ being the set of stochastic deteriorations that are equivalent to a finite sequence of simple CIMRIs (CDMRIs). 
Proof of Theorem 2. Given $E[\tilde{\epsilon} \mid \tilde{w}]=0$,

$$
\phi^{\prime}(\alpha)=E \tilde{\epsilon} u_{1}(\tilde{w}+\alpha \tilde{\epsilon}, \tilde{y})=E_{\tilde{w}} E\left[\tilde{\epsilon} u_{1}(\tilde{w}+\alpha \tilde{\epsilon}, \tilde{y}) \mid \tilde{w}\right]=E_{\tilde{w}} \operatorname{cov}\left[\tilde{\epsilon}, u_{1}(\tilde{w}+\alpha \tilde{\epsilon}, \tilde{y}) \mid \tilde{w}\right]
$$

(i) Given that $u_{12}<(>) 0$ and $E[\tilde{\epsilon} \mid \tilde{y} \leq y, \tilde{w}=w] \leq(\geq) E[\tilde{\epsilon} \mid \tilde{w}=w]$ for all $y$ and $w$ and there exists $Z_{w} \subset[-a, a]$ such that $P\left[\tilde{w}^{-1}\left(Z_{w}\right)\right]>0$ and, for $w \in Z_{w}$, the inequality is strict for $y \in Z_{y(w)} \subset[-a, a]$ where $P\left[\tilde{y}^{-1}\left(Z_{y(w)}\right)\right]>0$, Lemma 1 implies $\operatorname{cov}\left[\tilde{\epsilon}, u_{1}(\tilde{w}, \tilde{y}) \mid \tilde{w}=w\right]<0$ for all $w \in Z_{w}$ and thus

$$
\phi^{\prime}(0)=E_{\tilde{w}} E\left[\tilde{\epsilon} u_{1}(\tilde{w}, \tilde{y}) \mid \tilde{w}\right]=E_{\tilde{w}} \operatorname{cov}\left[\tilde{\epsilon}, u_{1}(\tilde{w}, \tilde{y}) \mid \tilde{w}\right]<0 .
$$

Since $u_{11}<0$ implies $\phi^{\prime \prime}(\alpha)=E \tilde{\epsilon}^{2} u_{11}(\tilde{w}+\alpha \tilde{\epsilon}, \tilde{y})<0$, we then have $\phi^{\prime}(\alpha)<0$ for $\alpha \geq 0$.

Conversely, given $\phi^{\prime}(0)=E_{\tilde{w}} \operatorname{cov}\left[\tilde{\epsilon}, u_{1}(\tilde{w}, \tilde{y}) \mid \tilde{w}\right]$, if $E[\tilde{x} \mid \tilde{y} \leq y, \tilde{w}=w] \leq(\geq) E[\tilde{x} \mid \tilde{w}=w]$ for all $y$ and $w$ but there does not exist $Z_{w} \subset[-a, a]$ such that $P\left[\tilde{w}^{-1}\left(Z_{w}\right)\right]>0$ and, for $w \in Z_{w}$, the inequality is strict for $y \in Z_{y(w)} \subset[-a, a]$ where $P\left[\tilde{y}^{-1}\left(Z_{y(w)}\right)\right]>0$, then $\phi^{\prime}(0)=0$. If for $\tilde{w}=w \in J \subset[-a, a]$, $\tilde{\epsilon}$ is not non-negative (non-positive) expectation dependent on $\tilde{y}$, by Lemma 1 , we can find $u$ such that $u_{11}<0$ and $u_{12}<(>) 0$ and $\operatorname{cov}\left[\tilde{\epsilon}, u_{1}(\tilde{w}, \tilde{y}) \mid \tilde{w}=w\right]>0$ for $w \in J$ but $\left(u_{12}(\right.$,$) is such that)$ $\left|\operatorname{cov}\left[\tilde{\epsilon}, u_{1}(\tilde{w}, \tilde{y}) \mid \tilde{w}=w\right]\right|$ is arbitrarily close to 0 for $w \notin J$, which implies $\phi^{\prime}(0)>0$ and thus we cannot have $\phi^{\prime}(\alpha)<0$ for $\alpha \geq 0$.

(ii) Analogous to (i).

(iii) Given $\phi^{\prime}(0)=E_{\tilde{w}} \operatorname{cov}\left[\tilde{\epsilon}, u_{1}(\tilde{w}, \tilde{y}) \mid \tilde{w}\right]$, the sufficiency of zero expectation dependence is obvious in view of the proof of (i).

To prove the necessity, if for $\tilde{w} \in J, \tilde{\epsilon}$ is not zero expectation dependent on $\tilde{y}$, by Lemma 1 , we can find $u$ such that $u_{11}<0$ and $u_{12}<0$ and $\operatorname{cov}\left[\tilde{\epsilon}, u_{1}(\tilde{w}, \tilde{y}) \mid \tilde{w}\right]>0$ (or $\left.<0\right)$ for $\tilde{w} \in J$ but $\left(u_{12}(\right.$,$) is$ such that) $\left|\operatorname{cov}\left[\tilde{\epsilon}, u_{1}(\tilde{w}, \tilde{y}) \mid \tilde{w}\right]\right|$ is arbitrarily close to 0 for $w \notin J$, which implies $\phi^{\prime}(0) \neq 0$ and thus we cannot have $\phi^{\prime}(\alpha)<0$ for $\alpha>0$ and $\phi^{\prime}(\alpha)>0$ for $\alpha<0$. (The case of $u_{11}<0$ and $u_{12}>0$ follows analogously.)

(iv) The sufficiency of of $u_{11} \leq 0$ and $u_{12} \leq(\geq) 0$ can be proved analogously to (i).

The necessity of $u_{11} \leq 0$ and $u_{12} \leq(\geq) 0$ is implied by Theorem 2 in Finkelshtain et. al. (1999) since $\phi^{\prime}(\alpha) \leq 0$ for $\alpha \geq 0$ and $E[\tilde{\epsilon} \mid \tilde{y} \leq y, \tilde{w}=w] \leq(\geq) E[\tilde{\epsilon} \mid \tilde{w}=w]$ for all $w$ and $y$ implies that $E u(\bar{w}, \tilde{y}) \geq E u(\bar{w}+\tilde{\epsilon}, \tilde{y})$ for $\tilde{\epsilon}, \tilde{w}$ and $\tilde{y}$ such that $\tilde{w}$ is degenerate and $E[\tilde{\epsilon} \mid \tilde{y}=y]$ is non-decreasing in $y$. 
(v) Analogous to (iv).

\section{Proof of Theorem 3.}

$[($ ii $) \Longleftrightarrow($ iii $)]$

Define $\Gamma(x, y) \equiv v(x, y)-\lambda u(x, y)$. Differentiating, we obtain $\Gamma_{1}(x, y)=v_{1}(x, y)-\lambda u_{1}(x, y) \leq 0$, $\Gamma_{11}(x, y)=v_{11}(x, y)-\lambda u_{11}(x, y) \leq 0$, and $\Gamma_{12}(x, y)=v_{12}(x, y)-\lambda u_{12}(x, y) \leq(\geq) 0$.

Conversely, given $v(x, y)=\lambda u(x, y)+\Gamma(x, y) \Longleftrightarrow \Gamma(x, y)=-\lambda u(x, y)+v(x, y), \Gamma_{1} \leq 0, \Gamma_{11} \leq 0$, and $\Gamma_{12} \leq(\geq) 0$ clearly imply respectively $\lambda u_{1}(x, y) \geq v_{1}(x, y), \lambda u_{11}(x, y) \geq v_{11}(x, y)$, and $\lambda u_{12}(x, y) \geq(\leq) v_{12}(x, y)$.

$[(i i i) \Rightarrow(\mathrm{i})]$

$$
\begin{aligned}
& \operatorname{Ev}(\tilde{x}-\pi, \tilde{y}) \\
= & E \lambda u(\tilde{x}-\pi, \tilde{y})+E \Gamma(\tilde{x}-\pi, \tilde{y}) \\
\geq & E \lambda u(\tilde{x}-\pi, \tilde{y})+E \Gamma\left(\tilde{x}^{\prime}-\pi, \tilde{y}\right) \\
\geq & E \lambda u\left(\tilde{x}^{\prime}, \tilde{y}\right)+E \Gamma\left(\tilde{x}^{\prime}, \tilde{y}\right) \\
= & E v\left(\tilde{x}^{\prime}, \tilde{y}\right)
\end{aligned}
$$

where the first inequality is due to the fact that $F_{\tilde{x}^{\prime}-\pi, \tilde{y}}$ being a CIMRI (CDMRI) of $F_{\tilde{x}-\pi, \tilde{y}}, \Gamma_{11} \leq 0$ and $\Gamma_{12} \leq(\geq) 0$ imply $E \Gamma\left(\tilde{x}^{\prime}-\pi, \tilde{y}\right) \leq E \Gamma(\tilde{x}-\pi, \tilde{y})$, and the second inequality is due to $\Gamma_{1} \leq 0$. $\left[(i) \Rightarrow(\right.$ iv) $]$ Since, by Theorem 2, $F_{\tilde{w}+\alpha_{2} \tilde{\epsilon}, \tilde{y}}$ is a CIMRI (CDMRI) of $F_{\tilde{w}+\alpha_{1} \tilde{\epsilon}, \tilde{y}}$ if $\alpha_{2}>\alpha_{1} \geq 0$ and $\tilde{w}$, $\tilde{\epsilon}$, and $\tilde{y}$ are such that $E[\tilde{\epsilon} \mid \tilde{y} \leq y, \tilde{w}=x] \leq(\geq) E[\tilde{\epsilon} \mid \tilde{w}=w]=0$ for all $w$ and $y$, (i) clearly implies $\pi^{v} \geq \pi^{u}$

$[(\mathrm{iv}) \Rightarrow(\mathrm{iii})]$ Let $\tilde{w}=w_{1}$ with probability $p, \tilde{w}=w_{0}$ with probability $1-p$, and $(\tilde{\epsilon}, \tilde{y})=\left(0, y_{0}\right)$ if $\tilde{w}=w_{0}$ and $(\tilde{\epsilon}, \tilde{y})$ is a $50: 50$ chance of $\left(-\epsilon, y_{1}\right)$ or $\left(\epsilon, y_{2}\right)$ if $\tilde{w}=w_{1} . \pi^{u}$ and $\pi^{v}$ are given by

$$
\begin{aligned}
& \frac{1}{2} p\left[u\left(w_{1}-\epsilon, y_{1}\right)+u\left(w_{1}+\epsilon, y_{2}\right)\right]+(1-p) u\left(w_{0}, y_{0}\right)=\frac{1}{2} p\left[u\left(w_{1}-\pi^{u}, y_{1}\right)+u\left(w_{1}-\pi^{u}, y_{2}\right)\right]+(1-p) u\left(w_{0}-\pi^{u}, y_{0}\right) \\
& \frac{1}{2} p\left[v\left(w_{1}-\epsilon, y_{1}\right)+v\left(w_{1}+\epsilon, y_{2}\right)\right]+(1-p) v\left(w_{0}, y_{0}\right)=\frac{1}{2} p\left[v\left(w_{1}-\pi^{v}, y_{1}\right)+v\left(w_{1}-\pi^{v}, y_{2}\right)\right]+(1-p) v\left(w_{0}-\pi^{v}, y_{0}\right)
\end{aligned}
$$


Differentiation and simple algebraic manipulations yield

$$
\begin{gathered}
\left.\frac{d \pi^{u}}{d \epsilon}\right|_{\epsilon=0}=-\frac{\frac{1}{2} p\left[-u_{1}\left(w_{1}, y_{1}\right)+u_{1}\left(w_{1}, y_{2}\right)\right]}{\frac{1}{2} p\left[u_{1}\left(w_{1}, y_{1}\right)+u_{1}\left(w_{1}, y_{2}\right)\right]+(1-p) u_{1}\left(w_{0}, y_{0}\right)} \\
\left.\frac{d \pi^{v}}{d \epsilon}\right|_{\epsilon=0}=-\frac{\frac{1}{2} p\left[-v_{1}\left(w_{1}, y_{1}\right)+v_{1}\left(w_{1}, y_{2}\right)\right]}{\frac{1}{2} p\left[v_{1}\left(w_{1}, y_{1}\right)+v_{1}\left(w_{1}, y_{2}\right)\right]+(1-p) v_{1}\left(w_{0}, y_{0}\right)} \\
+\left.\frac{\left.\frac{d^{2} \pi^{u}}{d \epsilon^{2}}\right|_{\epsilon=0}=-\frac{\frac{1}{2} p\left[u_{11}\left(w_{1}, y_{1}\right)+u_{11}\left(w_{1}, y_{2}\right)\right]}{\frac{1}{2} p\left[u_{1}\left(w_{1}, y_{1}\right)+u_{1}\left(w_{1}, y_{2}\right)\right]+(1-p) u_{1}\left(w_{0}, y_{0}\right)}}{\left.\frac{\left.d_{1}\left(w_{1}, y_{1}\right)+u_{1}\left(w_{1}, y_{2}\right)\right]\left\{\frac{1}{2} p\left[u_{11}\left(w_{1}, y_{1}\right)+u_{11}\left(w_{1}, y_{2}\right)\right]+(1-p) u_{11}\left(w_{0}, y_{0}\right)\right\}}{d \epsilon^{2}}\right|_{\epsilon=0}=-\frac{1}{\frac{1}{2} p\left[v_{1}\left(w_{1}, y_{1}\right)+v_{1}\left(w_{1}, y_{2}\right)\right]+(1-p) v_{1}\left(w_{0}, y_{0}\right)}} \frac{\left.d u_{1}\right)}{d \epsilon}\right|_{\epsilon=0} \\
+\left.\frac{\frac{1}{2} p\left[-v_{1}\left(w_{1}, y_{1}\right)+v_{1}\left(w_{1}, y_{2}\right)\right]\left\{\frac{1}{2} p\left[v_{11}\left(w_{1}, y_{1}\right)+v_{11}\left(w_{1}, y_{2}\right)\right]+(1-p) v_{11}\left(w_{0}, y_{0}\right)\right\}}{\left[\frac{1}{2} p\left[v_{1}\left(w_{1}, y_{1}\right)+v_{1}\left(w_{1}, y_{2}\right)\right]+(1-p) v_{1}\left(w_{0}, y_{0}\right)\right]^{2}} \frac{d \pi^{v}}{d \epsilon}\right|_{\epsilon=0}
\end{gathered}
$$

If $y_{1}<(>) y_{2}$ and thus conditional on $\tilde{w}, \tilde{\epsilon}$ is non-negative (non-positive) expectation dependent on $\tilde{y}, \pi^{v} \geq \pi^{u}$ for all $\epsilon>0$ implies that

$$
\frac{\frac{1}{2} p\left[v_{1}\left(w_{1}, y_{1}\right)-v_{1}\left(w_{1}, y_{2}\right)\right]}{\frac{1}{2} p\left[v_{1}\left(w_{1}, y_{1}\right)+v_{1}\left(w_{1}, y_{2}\right)\right]+(1-p) v_{1}\left(w_{0}, y_{0}\right)} \geq \frac{\frac{1}{2} p\left[u_{1}\left(w_{1}, y_{1}\right)-u_{1}\left(w_{1}, y_{2}\right)\right]}{\frac{1}{2} p\left[u_{1}\left(w_{1}, y_{1}\right)+u_{1}\left(w_{1}, y_{2}\right)\right]+(1-p) u_{1}\left(w_{0}, y_{0}\right)}
$$

or equivalently

$$
\begin{gathered}
p\left[v_{1}\left(w_{1}, y_{1}\right) u_{1}\left(w_{1}, y_{2}\right)-v_{1}\left(w_{1}, y_{2}\right) u_{1}\left(w_{1}, y_{1}\right)\right]+ \\
(1-p)\left[v_{1}\left(w_{1}, y_{1}\right)-v_{1}\left(w_{1}, y_{2}\right)\right] u_{1}\left(w_{0}, y_{0}\right)-(1-p)\left[u_{1}\left(w_{1}, y_{1}\right)-u_{1}\left(w_{1}, y_{2}\right)\right] v_{1}\left(w_{0}, y_{0}\right) \geq 0
\end{gathered}
$$

which, since $p$ can be arbitrarily small, implies

$$
\frac{v_{1}\left(w_{1}, y_{1}\right)-v_{1}\left(w_{1}, y_{2}\right)}{v_{1}\left(w_{0}, y_{0}\right)} \geq \frac{u_{1}\left(w_{1}, y_{1}\right)-u_{1}\left(w_{1}, y_{2}\right)}{u_{1}\left(w_{0}, y_{0}\right)}
$$

which, in turn, implies for all $\left(w_{0}, y_{0}\right)$ and $\left(w_{1}, y_{1}\right)$

$$
-\frac{v_{12}\left(w_{1}, y_{1}\right)}{v_{1}\left(w_{0}, y_{0}\right)} \geq-\frac{u_{12}\left(w_{1}, y_{1}\right)}{u_{1}\left(w_{0}, y_{0}\right)} \quad\left(\frac{v_{12}\left(w_{1}, y_{1}\right)}{v_{1}\left(w_{0}, y_{0}\right)} \geq \frac{u_{12}\left(w_{1}, y_{1}\right)}{u_{1}\left(w_{0}, y_{0}\right)}\right)
$$

Thus, letting

$$
\lambda \equiv \sup \left\{\frac{v_{1}(w, y)}{u_{1}(w, y)}:(w, y) \in[a, b]^{2}\right\}
$$


for all $\left(w_{0}, y_{0}\right)$ and $\left(w_{1}, y_{1}\right)$,

$$
\frac{v_{12}\left(w_{1}, y_{1}\right)}{u_{12}\left(w_{1}, y_{1}\right)} \geq \lambda \geq \frac{v_{1}\left(w_{0}, y_{0}\right)}{u_{1}\left(w_{0}, y_{0}\right)}
$$

Since

$$
\left.\frac{d \pi^{u}}{d \epsilon}\right|_{\epsilon=0} \rightarrow 0 \text { and }\left.\frac{d \pi^{v}}{d \epsilon}\right|_{\epsilon=0} \rightarrow 0 \text { as } y_{2} \rightarrow y_{1},
$$

$\pi^{v} \geq \pi^{u}$ for all $\epsilon>0$ also implies that for all $w_{0}, w_{1}, y_{0}, y_{1}, p$,

$-\frac{\frac{1}{2} p\left[v_{11}\left(w_{1}, y_{1}\right)+v_{11}\left(w_{1}, y_{2}\right)\right]}{\frac{1}{2} p\left[v_{1}\left(w_{1}, y_{1}\right)+v_{1}\left(w_{1}, y_{2}\right)\right]+(1-p) v_{1}\left(w_{0}, y_{0}\right)} \geq-\frac{\frac{1}{2} p\left[u_{11}\left(w_{1}, y_{1}\right)+u_{11}\left(w_{1}, y_{2}\right)\right]}{\frac{1}{2} p\left[u_{1}\left(w_{1}, y_{1}\right)+u_{1}\left(w_{1}, y_{2}\right)\right]+(1-p) u_{1}\left(w_{0}, y_{0}\right)}$

or

$$
\frac{v_{11}\left(w_{1}, y_{1}\right)+v_{11}\left(w_{1}, y_{2}\right)}{u_{11}\left(w_{1}, y_{1}\right)+u_{11}\left(w_{1}, y_{2}\right)} \geq \frac{\frac{1}{2} p\left[v_{1}\left(w_{1}, y_{1}\right)+v_{1}\left(w_{1}, y_{2}\right)\right]+(1-p) v_{1}\left(w_{0}, y_{0}\right)}{\frac{1}{2} p\left[u_{1}\left(w_{1}, y_{1}\right)+u_{1}\left(w_{1}, y_{2}\right)\right]+(1-p) u_{1}\left(w_{0}, y_{0}\right)}
$$

clearly for all $\left(w_{0}, y_{0}\right)$ and $\left(w_{1}, y_{1}\right)$

$$
\frac{v_{11}\left(w_{1}, y_{1}\right)}{u_{11}\left(w_{1}, y_{1}\right)} \geq \lambda \geq \frac{v_{1}\left(w_{0}, y_{0}\right)}{u_{1}\left(w_{0}, y_{0}\right)}
$$

since if there exist $\left(w_{0}^{\prime}, y_{0}^{\prime}\right)$ and $\left(w_{1}^{\prime}, y_{1}^{\prime}\right)$ such that

$$
\frac{v_{1}\left(w_{0}^{\prime}, y_{0}^{\prime}\right)}{u_{1}\left(w_{0}^{\prime}, y_{0}^{\prime}\right)}>\frac{v_{11}\left(w_{1}^{\prime}, y_{1}^{\prime}\right)}{u_{11}\left(w_{1}^{\prime}, y_{1}^{\prime}\right)}
$$

then for $p$ sufficiently small we have a contradiction.

$[($ iv $) \Longleftrightarrow(\mathrm{v})]$

Defining $\pi^{u}(\delta, \alpha, \tilde{w}, \tilde{\epsilon}, \tilde{y})$ and $\pi^{v}(\delta, \alpha, \tilde{w}, \tilde{\epsilon}, \tilde{y})$ respectively by the solutions for $\pi^{u}$ and $\pi^{v}$ to

$$
E u\left(\tilde{w}+(\alpha+\delta) \tilde{\epsilon}+\pi^{u}, \tilde{y}\right)=E u(\tilde{w}+\alpha \tilde{\epsilon}, \tilde{y}) \text { and } E v\left(\tilde{w}+(\alpha+\delta) \tilde{\epsilon}+\pi^{v}, \tilde{y}\right)=E v(\tilde{w}+\alpha \tilde{\epsilon}, \tilde{y}) \text {, }
$$

we have

$$
\left.\frac{\partial \pi^{u}}{\partial \delta}\right|_{\delta=0}=-\frac{E \tilde{\epsilon} u_{1}(\tilde{w}+\alpha \tilde{\epsilon}, \tilde{y})}{E u_{1}(\tilde{w}+\alpha \tilde{\epsilon}, \tilde{y})} \text { and }\left.\frac{\partial \pi^{v}}{\partial \delta}\right|_{\delta=0}=-\frac{E \tilde{\epsilon} v_{1}(\tilde{w}+\alpha \tilde{\epsilon}, \tilde{y})}{E v_{1}(\tilde{w}+\alpha \tilde{\epsilon}, \tilde{y})}
$$

(iv) then implies and is implied by

$$
-\frac{E \tilde{\epsilon} u_{1}(\tilde{w}+\alpha \tilde{\epsilon}, \tilde{y})}{E u_{1}(\tilde{w}+\alpha \tilde{\epsilon}, \tilde{y})} \leq-\frac{E \tilde{\epsilon} v_{1}(\tilde{w}+\alpha \tilde{\epsilon}, \tilde{y})}{E v_{1}(\tilde{w}+\alpha \tilde{\epsilon}, \tilde{y})}
$$

for all $\alpha, \tilde{w}, \tilde{\epsilon}$, and $\tilde{y}$ such that $E[\tilde{\epsilon} \mid \tilde{y} \leq y, \tilde{w}=x] \leq(\geq) E[\tilde{\epsilon} \mid \tilde{w}=w]=0$ for all $w$ and $y$. 
Since the first-order conditions for $\alpha_{u}^{*}$ and $\alpha_{v}^{*}$ are given by,

$$
E \tilde{x} u_{1}\left(\tilde{w}+\alpha_{u}^{*} \tilde{x}, \tilde{y}\right)=E(E[\tilde{x}]+\tilde{\epsilon}) u_{1}\left(\tilde{w}+\alpha_{u}^{*} \tilde{x}, \tilde{y}\right)=0
$$

and

$$
E \tilde{x} v_{1}\left(\tilde{w}+\alpha_{v}^{*} \tilde{x}, \tilde{y}\right)=E(E[\tilde{x}]+\tilde{\epsilon}) v_{1}\left(\tilde{w}+\alpha_{v}^{*} \tilde{x}, \tilde{y}\right)=0
$$

or equivalently

$$
-\frac{E \tilde{\epsilon} u_{1}\left(\tilde{w}+\alpha_{u}^{*} \tilde{x}, \tilde{y}\right)}{E u_{1}\left(\tilde{w}+\alpha_{u}^{*} \tilde{x}, \tilde{y}\right)}=E[\tilde{x}]=-\frac{E \tilde{\epsilon} v_{1}\left(\tilde{w}+\alpha_{v}^{*} \tilde{x}, \tilde{y}\right)}{E v_{1}\left(\tilde{w}+\alpha_{v}^{*} \tilde{x}, \tilde{y}\right)},
$$

(iv) clearly implies that

$$
-\frac{E \tilde{\epsilon} v_{1}\left(\tilde{w}+\alpha_{u}^{*} \tilde{x}, \tilde{y}\right)}{E v_{1}\left(\tilde{w}+\alpha_{u}^{*} \tilde{x}, \tilde{y}\right)} \geq E[\tilde{x}]
$$

$v_{11} \leq 0$ then implies $\alpha_{v}^{*} \leq \alpha_{u}^{*}$.

Conversely, if there exist $\alpha^{\prime}, \tilde{w}^{\prime}, \tilde{\epsilon}^{\prime}$, and $\tilde{y}^{\prime}$ such that $E\left[\tilde{\epsilon}^{\prime} \mid \tilde{w}^{\prime}\right]=0$ and conditional on $\tilde{w}^{\prime}, \tilde{\epsilon}^{\prime}$ is non-negative (non-positive) expectation dependent on $\tilde{y}^{\prime}$ and

$$
-\frac{E \tilde{\epsilon}^{\prime} u_{1}\left(\tilde{w}^{\prime}+\alpha^{\prime} \tilde{\epsilon}^{\prime}, \tilde{y}^{\prime}\right)}{E u_{1}\left(\tilde{w}^{\prime}+\alpha^{\prime} \tilde{\epsilon}^{\prime}, \tilde{y}^{\prime}\right)}>-\frac{E \tilde{\epsilon}^{\prime} v_{1}\left(\tilde{w}^{\prime}+\alpha^{\prime} \tilde{\epsilon}^{\prime}, \tilde{y}^{\prime}\right)}{E v_{1}\left(\tilde{w}^{\prime}+\alpha^{\prime} \tilde{\epsilon}^{\prime}, \tilde{y}^{\prime}\right)}
$$

letting

$$
E\left[\tilde{x}^{\prime}\right] \equiv-\frac{E \tilde{\epsilon}^{\prime} u_{1}\left(\tilde{w}^{\prime}+\alpha^{\prime} \tilde{\epsilon}^{\prime}, \tilde{y}^{\prime}\right)}{E u_{1}\left(\tilde{w}^{\prime}+\alpha^{\prime} \tilde{\epsilon}^{\prime}, \tilde{y}^{\prime}\right)}, \tilde{x}^{\prime} \equiv E\left[\tilde{x}^{\prime}\right]+\tilde{\epsilon}^{\prime}, \tilde{w}_{0} \equiv \tilde{w}^{\prime}-\alpha^{\prime} E\left[\tilde{x}^{\prime}\right]
$$

we have

$$
\begin{gathered}
E\left[\tilde{x}^{\prime}\right]=-\frac{E \tilde{\epsilon}^{\prime} u_{1}\left(\tilde{w}_{0}+\alpha^{\prime} \tilde{x}^{\prime}, \tilde{y}^{\prime}\right)}{E u_{1}\left(\tilde{w}_{0}+\alpha^{\prime} \tilde{x}^{\prime}, \tilde{y}^{\prime}\right)}=-\frac{E \tilde{\epsilon}^{\prime} u_{1}\left(\tilde{w}^{\prime}+\alpha^{\prime} \tilde{\epsilon}^{\prime}, \tilde{y}^{\prime}\right)}{E u_{1}\left(\tilde{w}^{\prime}+\alpha^{\prime} \tilde{\epsilon}^{\prime}, \tilde{y}^{\prime}\right)} \\
>-\frac{E \tilde{\epsilon}^{\prime} v_{1}\left(\tilde{w}^{\prime}+\alpha^{\prime} \tilde{\epsilon}^{\prime}, \tilde{y}^{\prime}\right)}{E v_{1}\left(\tilde{w}^{\prime}+\alpha^{\prime} \tilde{\epsilon}^{\prime}, \tilde{y}^{\prime}\right)}=-\frac{E \tilde{\epsilon}^{\prime} v_{1}\left(\tilde{w}_{0}+\alpha^{\prime} \tilde{x}^{\prime}, \tilde{y}^{\prime}\right)}{E v_{1}\left(\tilde{w}_{0}+\alpha^{\prime} \tilde{x}^{\prime}, \tilde{y}^{\prime}\right)}
\end{gathered}
$$

Given $v_{11} \leq 0$, this implies $\alpha_{u}^{*}\left(\tilde{w}_{0}, \tilde{x}^{\prime}, \tilde{y}^{\prime}\right)=\alpha^{\prime}<\alpha_{v}^{*}\left(\tilde{w}_{0}, \tilde{x}^{\prime}, \tilde{y}^{\prime}\right)$.

\section{Proof of Corollary 4.}

$[(i i) \Rightarrow($ iii $)]$ From (ii) we have that

$$
\frac{\partial}{\partial x} \ln u_{1}(x, y) \geq \lambda, \quad \frac{\partial}{\partial x} \ln \left(-u_{11}(x, y)\right) \leq \lambda, \quad \frac{\partial}{\partial x} \ln \left(-u_{12}(x, y)\right) \leq \lambda\left(\frac{\partial}{\partial x} \ln \left(u_{12}(x, y)\right) \leq \lambda\right)
$$


which implies that for $\delta>0$

$$
u_{1}(x+\delta, y) \geq e^{\lambda \delta} u_{1}(x, y), \quad u_{11}(x+\delta, y) \geq e^{\lambda \delta} u_{11}(x, y), \quad u_{12}(x+\delta, y) \geq(\leq) e^{\lambda \delta} u_{12}(x, y)
$$

Letting $\bar{\lambda}(\delta) \equiv e^{\lambda \delta}$ and $\Gamma(x, y ; \delta) \equiv u(x+\delta, y)-\bar{\lambda}(\delta) u(x, y)$ yields the desired result.

$[($ iii $) \Rightarrow($ ii $)]$

$\Gamma(x, y ; \delta)=u(x+\delta, y)-\bar{\lambda}(\delta) u(x, y), \Gamma_{1}(x, y ; \delta) \geq 0, \Gamma_{11}(x, y ; \delta) \geq 0$, and $\Gamma_{12}(x, y ; \delta) \geq(\leq) 0$ clearly imply

$$
u_{1}(x+\delta, y) \geq \bar{\lambda}(\delta) u_{1}(x, y), \quad u_{11}(x+\delta, y) \geq \bar{\lambda}(\delta) u_{11}(x, y), \quad u_{12}(x+\delta, y) \geq(\leq) \bar{\lambda}(\delta) \delta u_{12}(x, y) .
$$

For $\delta$ being small therefore,

$$
\begin{gathered}
u_{1}(x, y)+u_{11}(x, y) \delta \geq\left[\bar{\lambda}(0)+\bar{\lambda}^{\prime}(0) \delta\right] u_{1}(x, y) \Longleftrightarrow u_{11}(x, y) \geq \frac{\bar{\lambda}(0)+\bar{\lambda}^{\prime}(0) \delta-1}{\delta} u_{1}(x, y) \\
u_{11}(x, y)+u_{111}(x, y) \delta \geq\left[\bar{\lambda}(0)+\bar{\lambda}^{\prime}(0) \delta\right] u_{11}(x, y) \Longleftrightarrow u_{111}(x, y) \geq \frac{\bar{\lambda}(0)+\bar{\lambda}^{\prime}(0) \delta-1}{\delta} u_{11}(x, y) \\
u_{12}(x, y)+u_{112}(x, y) \delta \geq(\leq)\left[\bar{\lambda}(0)+\bar{\lambda}^{\prime}(0) \delta\right] u_{12}(x, y) \\
\Longleftrightarrow u_{112}(x, y) \geq(\leq) \frac{\bar{\lambda}(0)+\bar{\lambda}^{\prime}(0) \delta-1}{\delta} u_{12}(x, y)
\end{gathered}
$$

which implies $\bar{\lambda}(0)=1$ and letting $\lambda=\bar{\lambda}^{\prime}(0)$ yields the desired result.

\section{REFERENCES}

Atkinson, A. B. and F. Bourguignon (1982): "The Comparison of Multi-Dimensioned Distributions of Economic Status," Review of Economic Studies, 49, 183-201.

Dionne, George and Jingyuan Li (2014): "When Can Expected Utility Handle First-Order Risk Aversion?" Journal of Economic Theory 154, 403-422.

Doherty, Neil A. and Schlesinger, Harris (1983): "Optimal Insurance in Incomplete arkets," Journal of Political Economy, 91, 1045-1054.

Eeckhoudt, L., Gollier, C., Schlesinger, H. 1996. "Changes in background risk, temperance and risk taking behavior," Econometrica, 64, 683-689. 
Epstein, Larry G. and Tanny, Stephen M. (1980): “Increasing Generalized Correlation: A Definition and Some Economic Consequences," Canadian Journal of Economics, 13, 16-34.

Finkelshtain, Israel, Kella, Offer, and Scarsini, Marco (1999): "On Risk Aversion with Two Risks," Journal of Mathematical Economics, 31, 239-250.

Fishburn, P. C. and Vickson, R. G. (1978): "Theoretical Foundations of Stochastic Dominance," in G. Whitmore and M. Findlay, editors, Stochastic Dominance, 39-113. Lexington Books, Lexington, MA.

Gravel, Nicolas and Patrick Moyes (2012): “Ethically Robust Comparisons of Bidimensional Distributions with an Ordinal Attribute," Journal of Economic Theory, 147(4), 1384-1426.

Gollier, C. and Pratt, J. W., (1996): "Risk vulnerability and the tempering effect of background risk," Econometrica 64, 1109-1123.

Kimball, M. S., (1993): “Standard risk aversion," Econometrica, 61, 587-611.

Machina, Mark and Neilson, William (1987): "The Ross Measure of Risk Aversion: strengthening and Extension, Econometrica 55, 1139-1149.

Machina, Mark J. and John W. Pratt (1997): “Increasing Risk: Some Direct Constructions, " Journal of Risk and Uncertainty, 14, 103-127.

McFadden, David L. (1974): "On Some Facets of Betting," in Essays on Economic Behavior Under Uncertainty, ed. by M. Balch, D. McFadden, and S. Wu. Amsterdam: North Holland.

Muller, Alfred and Marco Scarsini (2012): "Fear of Loss, Inframodularity, and Transfers," Journal of Economic Theory, 147 (4), 1490-1500.

Levy, Haim and Paroush, Jacob (1974): “Toward Multivariate Efficiency Criteria," Journal of Economic Theory, 7, 129-142.

Li, Jingyuan (2011): "The Demand for a Risky Asset in the Presence of a Background Risk," Journal of Economic Theory 146, 372-391.

Pratt, John W. (1964): "Risk Aversion in the Small and the Large," Econometrica, 32 (1-2), 122-136. 
Pratt, John W. (1988): "Aversion to One Risk in the Presence of Others," Journal of Risk and Uncertainty, 1, 395-413.

Pratt, J. W. and Zeckhauser, R., (1987): “Proper risk aversion," Econometrica 55, 143-154.

Quah, John K.-H. (1981): "The Comparative Statics of Constrained Optimization Problems," Econometrica, 75(2), 401-431.

Ross, Stephen A. (1981): "Some Stronger Measures of Risk Aversion in the Small and the Large with Applications," Econometrica, 49(3), 621-638.

Rothschild, Michael and Joseph Stiglitz (1970): “Increasing risk I: A definition, Journal of Economic Theory, 2, 225-243.

Segal, Uzi and Avia Spivak (1990): "First Order versus Second Order Risk Aversion," Journal of Economic Theory, 51, 111-125.

Tsetlin, Ilia and Robert L. Winkler (2005): "Risky Choices and Correlated Background Risk," Management Science, 51(9), 1336-1345.

Wright, Randall (1987): “Expectation Dependence of Random Variables, with an Application in Portfolio Theory," Theory and Decision, 22, 111-124. 